\title{
Computing Radiative Heat Transfer Occurring in a Zone Fire Model
}

Glenn P. Forney 



\section{Computing Radiative Heat Transfer Occurring In A Zone Fire Model}

Glenn P. Forney

No vember 1991

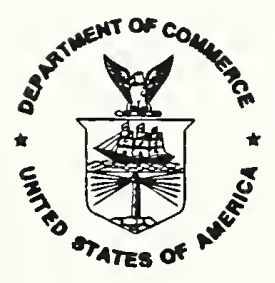

U.S. Department of Commerce

Robert A. Mosbacher, Secretary

National Institute of Standards and Technology

John W. Lyons, Director

Building and Fire Research Laboratory

Gaithersburg, MD 20899 



\section{Contents}

1 Introduction 1

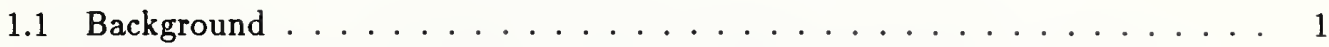

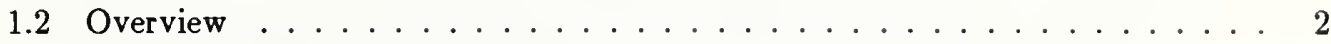

2 The Problem 3

2.1 N Wall Segment Radiation Exchange . . . . . . . . . . . . . . . . . . 3

2.1 .1 Modeling Assumptions . . . . . . . . . . . . . . . . . . . 3

2.1.2 Derivation of the Net Radiation Equations . . . . . . . . . . . 4

2.1.3 Heat Flux Striking a Wall Segment . . . . . . . . . . . . . . . 7

2.1 .4 Gas Absorbance . . . . . . . . . . . . . . . . . . 8

2.1.5 Configuration Factor Properties . . . . . . . . . . . . . . . 9

2.1 .6 Solid Angles . . . . . . . . . . . . . . . . . . . . . 14

2.1.7 Transmission Factors . . . . . . . . . . . . . . . . . 16

2.2 Two, Four, Ten Wall Segment Radiation Exchange . . . . . . . . . . . . 17

2.2 .1 Two-Wall Configuration Factors . . . . . . . . . . . . . 17

2.2 .2 Four-Wall Configuration Factors . . . . . . . . . . . . . 18

2.2.3 Ten-Wall Configuration Factors . . . . . . . . . . . . . . 20

3 Solving the Net Radiation Equations $\quad 25$

3.1 Solving The Net Radiation Equations Efficiently . . . . . . . . . . . 25

3.2 Algorithm for Calculating Four Wall Radiation Exchange . . . . . . . . 27

4 Computational Results 28

4.1 Checks . . . . . . . . . . . . . . . . . . . . . . . 28

4.2 Timings . . . . . . . . . . . . . . . . . . . . . . . . 29

4.3 Comparisons of RAD2 with RAD4 . . . . . . . . . . . . . 30

5 Conclusions $\quad 30$

Nomenclature $\quad 32$

$\begin{array}{ll}\text { References } & 35\end{array}$

$\begin{array}{ll}\text { Appendices } & 37\end{array}$

$\begin{array}{ll}\text { A Software Documentation } & 37\end{array}$

A.1 Using Subroutines RAD4 and RAD2 . . . . . . . . . . . . . 37

A.2 Using the Subroutine RAD10 . . . . . . . . . . . . . . . . . . 40

B Radiation Package Subroutine Structure - Who Calls Whom 40

$\begin{array}{ll}\text { C Subroutine Glossary } & 42\end{array}$ 


\section{List of Figures}

1 N-Wall Black Body Radiation Exchange . . . . . . . . . . . . . . . 6

2 Energy Distribution at the $k^{\prime}$ th Surface . . . . . . . . . . . . . 6

3 Energy Distribution Due to a Fire . . . . . . . . . . . . . . . 10

4 Energy Distribution Due to Wall Segment Emission . . . . . . . . . . . 10

5 Configuration Setup . . . . . . . . . . . . . . . . . 11

6 Configuration Factor Symmetries . . . . . . . . . . . . . . . . 13

7 Configuration Factor Setup For Pairs of Parallel and Perpendicular Rectangles 13

8 Solid Angle Setup . . . . . . . . . . . . . . . . . . 15

9 Solid Angles For Arbitrary Rectangles . . . . . . . . . . . . . . . . 15

10 Extended Floor, Ceiling Configuration Factor Setup . . . . . . . . . . . 19

11 Four Wall Configuration Factor Setup . . . . . . . . . . . . . . . . 19

12 Ten Wall Configuration Factor Setup . . . . . . . . . . . . . . . . 21

13 Ceiling, Upper Wall and Extended Ceiling Flux vs. Ceiling Temperature . . . 31

\section{List of Tables}

1 Radiative Heat Flux Striking the k'th Rectangular Wall Segment . . . . . . 8

2 Radiant Heat Absorbed by the Upper Layer . . . . . . . . . . . . . . . . . . 9

3 Radiant Heat Absorbed by the Lower Layer . . . . . . . . . . . . . . . . 11

4 Four Wall Radiation Algorithm Timings . . . . . . . . . . . . . . 29 


\title{
COMPUTING RADIATIVE HEAT TRANSFER OCCURRING IN A ZONE FIRE MODEL
}

\author{
Glenn P. Forney*
}

\begin{abstract}
Radiation, convection and conduction are the three mechanisms which a zone fire model must consider when calculating the heat transfer between fires, wall surfaces and room gases. Radiation dominates the other two modes of heat transfer in rooms where there are fires or hot smoke layers. The computational requirements of a radiation model can also easily dominate the work required to calculate other physical submodels in a zone fire model.

This report presents algorithms for efficiently computing the radiative heat exchange between four-wall surfaces, several fires and two interior gases. A two-wall and a ten-wall radiation model are also discussed. The structure of this radiation model is exploited to show that only a few configuration factors need to be calculated directly (two rather than 16 for the four-wall model and eight rather than 100 for the ten-wall model) and matrices needed to solve for the net radiative flux striking each surface are shown, after the appropriate transformation is taken, to be diagonally dominant. Iterative methods may then be used to solve the linear equations more efficiently than direct methods such as Gaussian elimination.

The radiation exchange algorithms are implemented as FORTRAN subroutines named RAD2, RAD4 and RAD10. These subroutines along with a test driver are available from the author.
\end{abstract}

\section{Introduction}

\subsection{Background}

Radiation is an important heat transfer mode to represent in a zone fire model due to the high temperatures attained in rooms with fires or hot smoke layers. It can easily dominate convective and conductive heat transfer. A radiative heat transfer calculation can also easily dominate the computation in any fire model. This is because radiation exchange is a global phenomena. Each portion of an enclosure interacts radiatively with every other portion that it 'sees'. It is therefore important to design radiation exchange algorithms that are efficient as well as accurate.

Most zone fire models use two wall segments to model radiation exchange. Harvard $\mathrm{V}$ [1], FIRST [2], BRI [3,4] and FAST [5] are some examples. FAST/FFM [6] on the other hand uses many surface segments in order to model the radiative interaction between

"Building and Fire Research Laboratory, National Institute of Standards and Technology, Gaithersburg, MD 20899, U.S.A. (gpfrnecfr6.cfr.nist.gov). 
wall surfaces and furniture elements. Harvard V and FIRST model the two wall segments as infinite parallel plates. BRI and FAST model the two wall segments as an extended floor and ceiling. The extended ceiling consists of the ceiling plus the four upper walls ${ }^{1}$. Similarly, the extended floor consists of the floor plus the four lower walls. The purpose of the work described in this report then is to enhance two wall radiation exchange algorithms by considering more wall segments. In particular for the four-wall case, this allows the ceiling, the upper wall segment, the lower wall segment and the floor to transfer radiant heat independently.

This report describes three algorithms for computing radiative heat transfer between the bounding surfaces of a compartment containing upper and lower layer gases and point source fires. The first algorithm uses two wall segments, the second uses four wall segments and the third uses ten wall segments. These algorithms each use the net radiation equation as described in Siegel and Howell [7, Chapter 17] which is based on Hottel's work in [8]. An enclosure is modeled with $N$ wall segments (for our case $N$ will be 2,4 or 10) and an interior gas. A two layer zone fire model, however, requires treatment of an enclosure with two uniform gases (the upper and lower layer). Hottel and Cohen [9] developed a method to handle this case by dividing an enclosure in to a number of wall and gas volume elements. Treatment of the fire and the interaction of the fire and gas layers with the walls is based upon the work of Yamada and Cooper $[10,11]$ on $\mathrm{N}$-wall radiation exchange models. They model the fire as a point source of heat radiating uniformly in all directions and use the Lambert-Beer law to model the interaction between heat emitting objects ${ }^{2}$ and gas layers.

The number of wall segments influences the accuracy and the efficiency of the radiation exchange model. The suitability of a two, four or ten wall radiation model then depends on the particular application. More wall segments are needed to model radiation exchange accurately in rooms with large temperature variations.

The two, four and ten wall algorithms are implemented as FORTRAN 77 subroutines named RAD2, RAD4 and RAD10. The routines, RAD2 and RAD10, take advantage of the modular structure of RAD4 by using a number of its routines. It should be pointed out that the computational requirements of a general $\mathrm{N}$-wall radiation model are too great for now to justify incorporating it into a zone fire model. By implementing the net radiation equation for particular $\mathrm{N}$ (two, four or ten walls), significant algorithmic speed increases were achieved by exploiting the structure of the simpler problems.

\subsection{Overview}

To put the two, four and ten wall radiation models into perspective, an $\mathrm{N}$-wall model is presented using the notation found in [7, Chapter 17]. Modeling assumptions are given and the net radiation equation is derived for the general $\mathrm{N}$-wall segment case. The $\mathrm{N}$-wall model is then adapted for two, four and ten walls. Two key results allow for significant algorithmic speed ups. First, it is shown that only eight configuration factors need to be calculated directly rather than 100 for the ten-wall case and two configuration factors rather than 16 for the four-wall case. This is important because the configuration factor calculation is one of the major bottlenecks in computing radiation heat exchange. Second, it is shown how a matrix involved in the solution of a set of simultaneous linear equations can be transformed into a diagonally dominant matrix. This is significant because an $o\left(N^{2}\right)$ rather than an

\footnotetext{
${ }^{1}$ The upper wall is the portion of a wall above the layer interface. Likewise, the lower wall is below the interface.

${ }^{2}$ fires, walls or gas layers for example
} 
$o\left(N^{3}\right)$ algorithm can then be used to solve the resulting linear system. This savings is more important for the ten-wall case (or larger) than the four-wall case. Some computational results are presented and the subroutines RAD2, RAD4 and RAD10 are documented. The structure of the subroutines used with RAD4 is documented and a short description of each subroutine used by RAD2, RAD4 and RAD10 is given.

\section{The Problem}

\subsection{N Wall Segment Radiation Exchange}

The $\mathrm{N}$-wall radiation model described in this section considers radiative heat transfer between wall segments, point-source fires and two gas layers. An enclosure or room is partitioned into $\mathrm{N}$ wall segments where each wall segment emits, reflects and absorbs radiant energy. The interior of the enclosure is partitioned into two volume elements; an upper and a lower layer. The problem then is to determine the net radiation flux emitted by each wall segment and the energy absorbed by each layer given the temperature and emittance of each wall segment and the temperature and absorptance of the two gas layers.

These calculations can be performed in conjunction with a zone fire model such as CFAST. Typically, the solution (wall temperatures, gas layer temperatures etc) is known at a given time $t$. The solution is then advanced to a new time, $t+\Delta t$. The calculated radiation fluxes along with convective fluxes are used as a boundary condition for an associated heat conduction problem in order to calculate wall temperatures. Gas layer energy absorption due to radiation contributes to the energy source terms of the associated zone fire modeling differential equations. The time step, $\Delta t$, must be chosen sufficiently small so that changes in wall temperatures are small over the duration of the time step.

\subsubsection{Modeling Assumptions}

The following assumptions are made in order to simplify the radiation heat exchange model and to make its calculation tractable.

iso-thermal Each gas layer and each wall segment is assumed to be at a uniform temperature. This assumption breaks down where wall segments meet.

equilibrium The wall segments and gas layers are assumed to be in a quasi-steady state. The wall and gas layer temperatures are assumed to change slowly over the duration of the time step of the associated differential equation.

fire source The fire is assumed to radiate uniformly in all directions giving off a fraction, $\chi$, of the total energy release rate to thermal radiation. This radiation is assumed to originate from a single point. Radiation feedback to the fire and radiation from the plume is not modeled. The plume could be modeled as a collection of point source fires ${ }^{3}$, however.

\footnotetext{
${ }^{3}$ RAD4, allows up to ten point source fires
} 
radiators

opacity

geometry
The radiation emitted from a wall surface, a gas and a fire is assumed to be diffuse and gray. In other words, the radiant fluxes emitted by these objects are independent of the direction and the wavelength. They can depend on temperature, however. Since both the emittances and the temperatures of wall segments are inputs to the radiation algorithms, it is assumed that the emittances are consistent with the corresponding wall temperatures. These assumptions ${ }^{4}$ allow us to infer that the emittance, $\epsilon$, absorbance, $\alpha$, and reflectance, $\rho$, are related via $\epsilon=\alpha=1-\rho$. A discussion of this assumption can be found in [12, p. 589-590].

The wall surfaces are assumed to be opaque. When radiation encounters a surface it is either reflected or absorbed. It is not transmitted through the surface. Equation (5) would have to be modified to account for the loss (or gain) of energy through semi-transparent surfaces.

Rooms or compartments are assumed to be rectangular boxes. Each wall is either perpendicular or parallel to every other wall. Radiation transfer through vent openings, doors, etc is ignored.

\subsubsection{Derivation of the Net Radiation Equations}

To put the general net radiation equations in perspective, it is first shown how to compute the radiation exchange between $\mathrm{N}$ black body surfaces. A general enclosure with $\mathrm{N}$ walls containing a transparent gas is depicted in Figure 1. Each wall, $k$, has a temperature $T_{k}$ and an area $A_{k}$. If each wall segment is a black-body then reflection terms can be ignored when computing the net radiation given off by each segment. This net radiation is the difference between the energy given off and the energy received at a wall segment as in

$$
A_{k} \Delta q^{\prime \prime}{ }_{k}=\sigma\left(A_{k} T_{k}^{4}-\sum_{j=1}^{N} A_{j} F_{j-k} T_{j}^{4}\right)
$$

where $F_{j-k}$ is the fraction of energy given off by wall $\mathrm{j}$ that is intercepted by segment $k$. The " notation is used to denote flux, ( $i e$ flow rate of mass or energy per unit area). The above equation can be simplified using the symmetry relation ${ }^{5}, A_{k} F_{k-j}=A_{j} F_{j-k}$, to obtain

$$
\Delta q^{\prime \prime}{ }_{k}=\sigma\left(T_{k}^{4}-\sum_{j=1}^{N} F_{k-j} T_{j}^{4}\right) .
$$

For the case of two infinite parallel plates, $\left(N=2, F_{2-1}=1\right)$, and $F_{2-2}=0$ the above equation reduces to the familiar formula for radiation exchange between two bodies,

$$
\begin{aligned}
& \Delta q^{\prime \prime}{ }_{2}=\sigma\left(T_{2}^{4}-T_{1}^{4}\right), \\
& \Delta q^{\prime \prime}{ }_{1}=\sigma\left(T_{1}^{4}-T_{2}^{4}\right)=-\Delta q^{\prime \prime}{ }_{2} .
\end{aligned}
$$

\footnotetext{
${ }^{4}$ Diffusivity implies that $\epsilon_{\lambda}=\alpha_{\lambda}$ for each wavelength $\lambda$ while the gray gas/surface assumption implies that $\varepsilon_{\lambda}$ is constant for all wavelengths

${ }^{5}$ This and other configuration factor properties will be discussed in section 2.1.5
} 
For the more general case, we must consider wall segments that are not black bodies and gas layers that are not transparent. For this case, the $k^{\prime}$ th surface emits an energy flux of $\sigma \epsilon_{k} T_{k}^{4}$ where $\epsilon_{k}<1$ and a reflectance term consisting of radiative fluxes emitted by other surfaces. The radiation exchange at the $k$ 'th surface is diagrammed in Figure 2. For each wall segment $\mathrm{k}$ from 1 to $N$ we must find a heat flux, $\Delta q^{\prime \prime}{ }_{k}$, such that

$$
\overbrace{A_{k} \sigma \epsilon_{k} T_{k}^{4}}^{\text {source }}+\overbrace{\left(1-\epsilon_{k}\right) q_{k}^{i n}}^{\text {reflected }}=\overbrace{q_{k}^{i n}}^{\text {incoming }}+\overbrace{A_{k} \Delta q^{\prime \prime}{ }_{k}}^{\text {net }}(k=1, \ldots, N) .
$$

Radiation exchange at each wall segment has surface, reflected, incoming and net radiation terms. The above equation then represents a system of linear equations that must be solved for $\Delta q^{\prime \prime}$ to determine the net fluxes given off by each surface. The set up and solution of this linear system is the bulk of the work required to implement the net radiation method of Siegel and Howell.

The outgoing radiation, $q_{k}^{\text {out }}$, consists of two components, gray-body surface radiation and in-coming radiation from other surfaces that is reflected. The incoming radiation, $q_{k}^{i n}$, is composed of gray-body surface radiation from other surfaces in the enclosure, radiating point-source fires and emission from two gas layers. The radiation leaving the $k$ 'th surface is given by

$$
q_{k}^{\text {out }}=A_{k} \sigma \epsilon_{k} T_{k}^{4}+\left(1-\epsilon_{k}\right) q_{k}^{\text {in }} .
$$

The radiation arriving at the $k$ 'th surface is

$$
q_{k}^{i n}=\sum_{j=1}^{N} q_{j}^{\text {out }} F_{j-k} \tau_{j-k}+c_{k}
$$

where $F_{j-k}$ is a configuration factor and is defined to be the fraction of energy leaving a surface $\mathrm{j}$ that is intercepted by surface $\mathrm{k}$. The term $\tau_{j-k}$ is a transmission factor, the fraction of energy that is transmitted unimpeded through the gas layer(s) between surfaces $j$ and $k$. The term, $c_{k}$, represents contributions due to other heat source such as heat emitting gas layers and point source fires. The computation of $F_{j-k}$ and $\tau_{j-k}$ is discussed in sections 2.1.5 and 2.1.7 respectively. The computation of $c_{k}$ is discussed in section 2.1.3.

The net radiation, $A_{k} \Delta q^{\prime \prime}{ }_{k}$, is the difference between the out-going and in-coming radiation terms. It is the amount of energy that must be supplied to the surface $\mathrm{k}$ in order to maintain a steady state, i.e. constant temperature wall segment. This is the net radiation that is being solved for. It is given by

$$
A_{k} \Delta q_{k}^{\prime \prime}=q_{k}^{\text {out }}-q_{k}^{\text {in }}
$$

and is described in [7, Chapters 8 and 17].

Equations (1)-(3) involve $3 N$ equations for the unknowns $q_{k}^{\text {out }}, q_{k}^{\text {in }}$ and $\Delta q^{\prime \prime}{ }_{k}$ for $k=$ $1, \cdots, N$. Siegel and Howell [7] find the net radiant flux $\Delta q^{\prime \prime}{ }_{k}$ for each of $N$ surfaces in an enclosure by solving equations (1), (2) and (3) for $\Delta q^{\prime \prime}{ }_{k}$ to yield the net radiation flux. The net radiation method was first developed by Hottell [8].

It should be noted that the net radiation method (solving for $\Delta q^{\prime \prime}{ }_{k}$ ) is superior numerically to the alternative of solving equations (1)-(3) for $q_{k}^{\text {out }}$. Though equivalent analytically, this "new" algorithm is inferior numerically to the net radiation method (solving for $\Delta q^{\prime \prime}{ }_{k}$ ) 


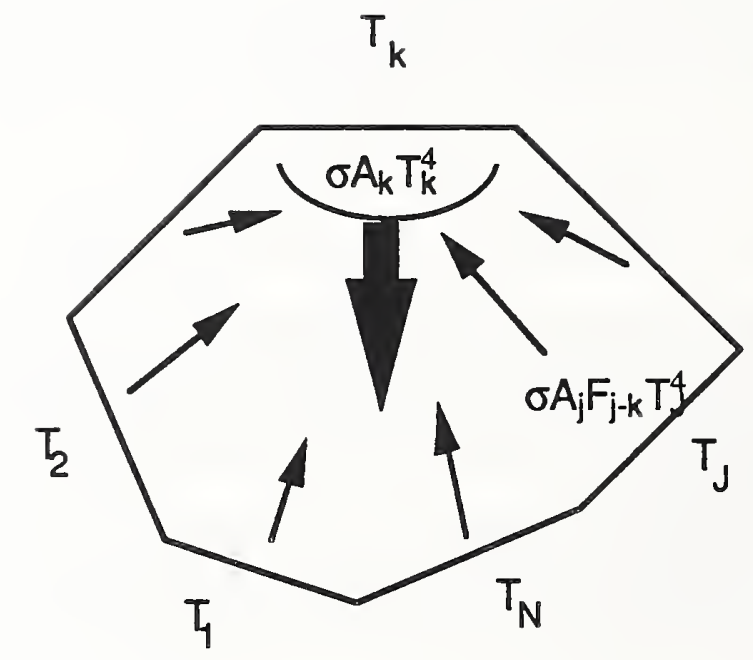

Figure 1: N-Wall Black Body Radiation Exchange

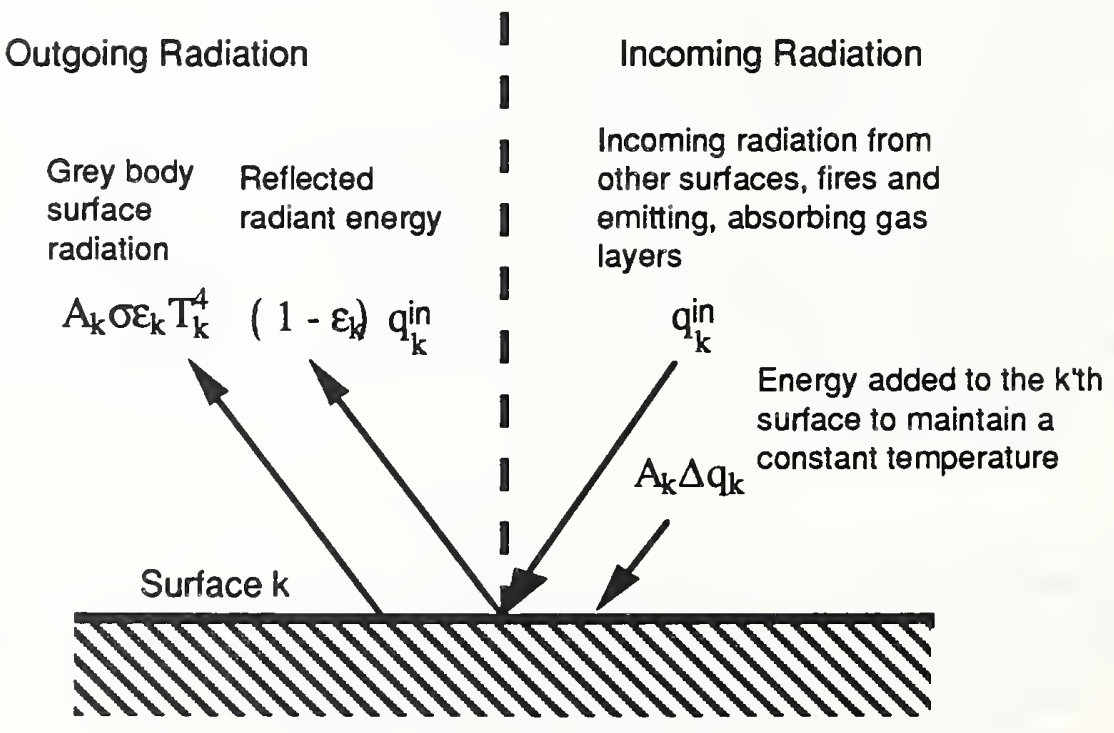

Figure 2: Energy Distribution at the k'th Surface 
because of the catastrophic cancellation errors introduced by the subtraction of nearly equal large numbers in equation (3). The same comment applies when solving for $q_{k}^{i n}$.

To solve by the net radiation method for $\Delta q^{\prime \prime}{ }_{k}$, first eliminate $q_{k}^{i n}$ in equations (1) and (2) by using equation (3) to obtain

$$
\begin{aligned}
q_{k}^{\text {out }} & =A_{k}\left(\sigma T_{k}^{4}-\frac{1-\epsilon_{k}}{\epsilon_{k}} \Delta q_{k}^{\prime \prime}\right) \\
q_{k}^{\text {out }}-A_{k} \Delta q^{\prime \prime}{ }_{k} & =\sum_{j=1}^{N} q_{j}^{\text {out }} F_{j-k} \tau_{j-k}+c_{k} .
\end{aligned}
$$

The term $q_{k}^{\text {out }}$ can be eliminated from the above equation by using (4) to obtain

$$
A_{k} \sigma T_{k}^{4}-A_{k} \frac{\Delta q_{k}^{\prime \prime}}{\epsilon_{k}}=\sum_{j=1}^{N}\left(A_{j} \sigma T_{j}^{4}-\frac{1-\epsilon_{j}}{\epsilon_{j}} A_{j} \Delta q_{j}\right) F_{j-k} \tau_{j-k}+c_{k}
$$

The area $A_{k}$ can be eliminated after rearranging terms and using the symmetry relationship $A_{k} F_{k-j}=A_{j} F_{j-k}$, to convert the above equation into the flux equation

$$
\frac{\Delta q^{\prime \prime}{ }_{k}}{\epsilon_{k}}-\sum_{j=1}^{N} \frac{1-\epsilon_{j}}{\epsilon_{j}} \Delta q^{\prime \prime}{ }_{j} F_{k-j} \tau_{j-k}=\sigma T_{k}^{4}-\sum_{j=1}^{N} \sigma T_{j}^{4} F_{k-j} \tau_{j-k}-\frac{c_{k}}{A_{k}} .
$$

Equation (5), listed in [7] as equation (17-20) is the net radiation equation. The algorithms used in this report use equations that are equivalent analytically to the net radiation equations but different (and superior) numerically. These modifications and their importance are discussed in section 3.1

\subsubsection{Heat Flux Striking a Wall Segment}

In general, every possible path between two wall segments should be considered in order to compute the total radiant heat transfer between these segments. This is not practical in a zone fire model due to the excessive computational costs. The approach taken here is to model this heat transfer using just one path. For a typical path there are four cases to consider. A path from wall segment $\mathrm{j}$ to $\mathrm{k}$ can start in either the upper or lower layer and may finish in either the upper or lower layer. A fraction, $\alpha=1-\tau$, of the energy encountering a layer is absorbed. The rest, $\tau$, passes through unimpeded. Table 1 gives formulas for the heat flux striking the $k$ 'th wall segment due to point source fires and heat emitting gas layers. These formulas are components of $c_{k}^{\prime \prime}$ which appear in equation (23). Subsequent sub-sections discuss how to compute the components of the equations in Table 1.

Heat Flux Striking a Wall Segment Due to a Point Source Fire The solid angle of a surface is the fraction of the total radiant energy released by the fire that strikes the given surface. The fire is assumed to radiate uniformly in all directions. In the case of a point source a solid angle is a configuration factor. If the gas layers are transparent then the flux striking the $k$ 'th surface due to the f'th fire is 
Table 1: Radiative Heat Flux Striking the k'th Rectangular Wall Segment

\begin{tabular}{|c|c|c|c|}
\hline \multirow[t]{2}{*}{ Path } & Fire & \multicolumn{2}{|c|}{ Gas Layer } \\
\hline & $q_{f-k}^{\prime \prime f i r e}$ & $q_{j-k}^{\prime \prime L, g a s}$ & $q_{j-k}^{\prime \prime}{ }_{j, g a s}$ \\
\hline in upper & $\tau_{f-k}^{U} \frac{\chi q_{f o f i a l}^{f i r e} \omega_{f-k}}{4 \pi A_{k}}$ & 0 & $F_{k-j} \sigma \alpha_{j-k}^{U} T_{U}^{4}$ \\
\hline from upper to lower & $\tau_{f-k}^{U} \tau_{f-k}^{L} \frac{\chi q_{\text {oftal }}^{f \text { ire }} \omega_{f-k}}{4 \pi A_{k}}$ & $F_{k-j} \sigma \alpha_{j-k}^{L} T_{L}^{4}$ & $F_{k-j} \sigma \alpha_{j-k}^{U} T_{U}^{4} \tau_{j-k}^{L}$ \\
\hline from lower to upper & $\tau_{f-k}^{L} \tau_{f-k}^{U} \frac{\chi q_{i \text { of alal }}^{f \text { fire }} w_{f-k}}{4 \pi A_{k}}$ & $F_{k-j} \sigma \alpha_{j-k}^{L} T_{L}^{4} \tau_{j-k}^{L}$ & $F_{k-j} \sigma \alpha_{j-k}^{U} T_{U}^{4}$ \\
\hline in lower & $\tau_{f-k}^{L} \frac{\chi q_{\text {total }}^{f\left(\cos _{j} w_{f-k}\right.}}{4 \pi A_{k}}$ & $F_{k-j} \sigma \alpha_{j-k}^{L} T_{L}^{4}$ & 0 \\
\hline
\end{tabular}

$$
q_{f-k}^{\prime \prime f i r e}=\frac{\chi q_{t o t a l}^{f i r e} \omega_{f-k}}{4 \pi A_{k}}
$$

The total energy release rate of the fire is $q_{\text {total }}^{\text {fire }}, \chi$ is the fraction of this energy that contributes to radiation, $\omega_{f-k} /\left(4 \pi A_{k}\right)$ is the fraction of the radiant energy leaving the $\mathrm{f}^{\prime}$ th fire that is intercepted by the $k$ 'th wall segment, ie a configuration factor. On the other hand, if the gas layers are not transparent then there are four cases to consider when calculating the heat transfer from a fire to a wall segment. The fire can be in the upper or lower layer and the surface can be in the upper or lower layer. Figure 3 shows how radiation from a fire is absorbed by each layer when the fire is in the lower layer and the surface $\mathrm{k}$ is in the upper layer. The other three cases are handled similarly. These four cases are summarized in the first column of Table 1. This column gives formulas for the flux striking a surface $k$ due to a point source fire.

Heat Flux Striking a Wall Segment Due to an Emitting Gas Layer The energy emitted by the i'th layer ( $i=$ upper, or $i=$ lower) along the $j-k$ 'th path is

$$
q_{j-k}^{\prime \prime, g a s}=\alpha_{j-k}^{i} \sigma T_{i}^{4}
$$

where $\alpha_{j-k}^{i}=1-\tau_{j-k}^{i}$. The emittance of the gas in this equation is the same as the absorptance due to the gray gas assumption. The computation of $\alpha$ and $\tau$ is discussed in section 2.1.7. Again four cases must be considered to calculate the flux striking a wall segment. The last column of Table 1 gives formulas for radiation striking the $k$ 'th wall segment due to gas layer heat emissions for each possible path.

\subsubsection{Gas Absorbance}

The net radiation leaving each surface, $\mathrm{k}$, is $\Delta q^{\prime \prime}{ }_{k}$. If the fire is ignored then the total energy added to the gas is

$$
\sum_{j=1}^{N} A_{j} \Delta q^{\prime \prime}{ }_{j}
$$


Table 2: Radiant Heat Absorbed by the Upper Layer

\begin{tabular}{|c|c|c|c|}
\hline $\begin{array}{l}\text { Path through the } \\
\text { Gas }\end{array}$ & $\begin{array}{l}\text { Due to Heat Emitting } \\
\text { Wall Surface } \\
q_{j-k}^{\text {out }}=A_{j} F_{j-k}\left(\sigma T_{j}^{4}-\right. \\
\left.\qquad \frac{1-\epsilon_{j}}{\epsilon_{j}} \Delta q^{\prime \prime}{ }_{j}\right)\end{array}$ & 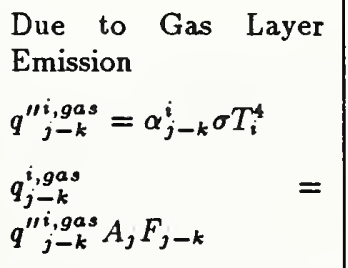 & $\begin{array}{l}\text { Due to Point Source } \\
\text { Fire } \\
q_{f-k}^{\prime \prime f i r e}=\frac{X q_{\text {total }}^{f i r e} \omega_{f-k}}{4 \pi A_{k}}\end{array}$ \\
\hline $\begin{array}{l}\text { from the upper to } \\
\text { either the lower or } \\
\text { upper layer }\end{array}$ & $q_{j-k}^{\text {out }} \alpha_{j-k}^{U}$ & $-q_{j-k}^{U, g a s}$ & $q_{f-k}^{\prime \prime f i r e} \alpha_{f-k}^{U}$ \\
\hline $\begin{array}{l}\text { from the lower to } \\
\text { the upper layer }\end{array}$ & $q_{j-k}^{\text {out }} \tau_{j-k}^{L} \alpha_{j-k}^{U}$ & $q_{j-k}^{L, g a s} \alpha_{j-k}^{U}-q_{j-k}^{U, g a s}$ & $q_{f-k}^{\prime \prime f i r e} \alpha_{f-k}^{U} \tau_{f-k}^{L}$ \\
\hline $\begin{array}{l}\text { from the lower to } \\
\text { the lower layer }\end{array}$ & 0 & 0 & 0 \\
\hline
\end{tabular}

If the gases are transparent then the above equation must sum to zero. This is a good check on RAD2, RAD4 and RAD10. The energy absorbed by the gas layers may be due to radiating wall segments, emission from other gas layers and radiation from fires. Tables 2 and 3 summarize the formulas used to compute gas layer energy gain/loss due to these phenomena. There are again four cases to consider, since an arbitrary path may start in either the lower or the upper layer and end in the lower or upper layer. Figure 4 illustrates the heat absorbed by the gas layers due to surface rectangle emission where the "from" wall segment is in the upper layer and the "to" wall segment is in the lower layer. The other three cases are handled similarly.

\subsubsection{Configuration Factor Properties}

A configuration factor, $F_{j-k}$, is the fraction of radiant energy leaving a surface $\mathrm{j}$ that is intercepted by a surface $\mathrm{k}$. The terms used to define a configuration factor are illustrated in Figure 5. Configuration factors, also called view or shape factors, are derived in numerous texts [13, Chapter 2], [12, Chapter 13], [7, Chapter 7]. This sub-section lists some configuration factor properties used later to reduce the number of required, direct configuration factor calculations and therefore to improve the efficiency of the radiation algorithm. Other configuration factors are calculated indirectly from these formulas using algebraic relationships based on equations $(7)-(11)$

The configuration factor from wall segment $j$ to wall segment $k$ is given by 


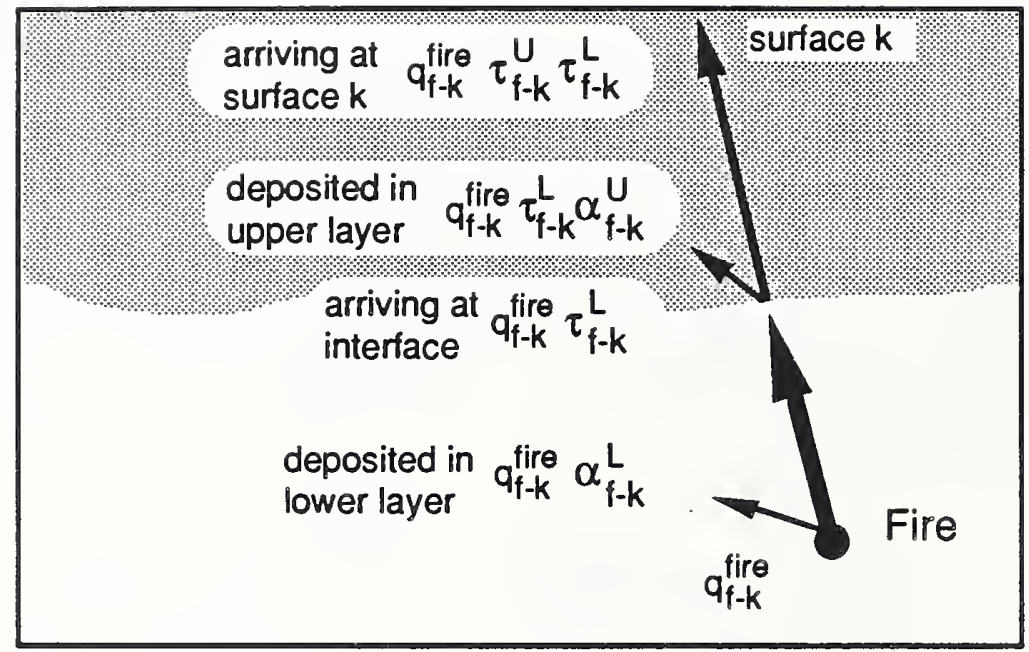

Figure 3: Energy Distribution Due to a Fire

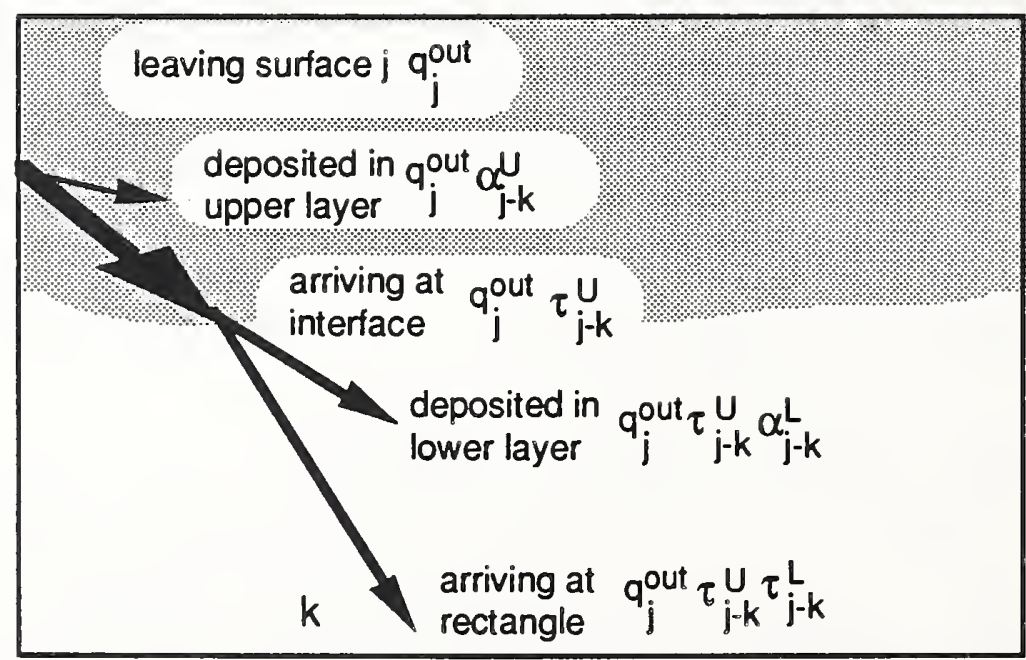

Figure 4: Energy Distribution Due to Wall Segment Emission 
Table 3: Radiant Heat Absorbed by the Lower Layer

\begin{tabular}{|c|c|c|c|}
\hline $\begin{array}{l}\text { Path through the } \\
\text { Gas }\end{array}$ & $\begin{array}{l}\text { Due to Heat Emitting } \\
\text { Wall Surface } \\
q_{j-k}^{\text {out }}=A j F_{j-k}\left(\sigma T_{j}^{4}-\right. \\
\left.\qquad \frac{1-\epsilon_{j}}{\epsilon_{j}} \Delta q_{j}^{\prime \prime}\right)\end{array}$ & 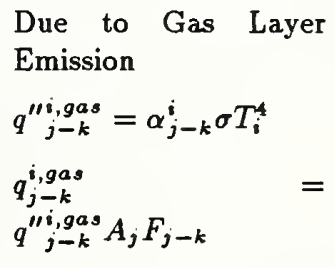 & $\begin{array}{l}\text { Due to Point Source } \\
\text { Fire } \\
q_{f-k}^{\prime \prime f i r e}=\frac{\chi q_{t o t a l}^{f(r e} w_{f-k}}{4 \pi A_{k}}\end{array}$ \\
\hline $\begin{array}{l}\text { from the lower to } \\
\text { either the lower or } \\
\text { upper layer }\end{array}$ & $q_{j-k}^{\text {out }} \alpha_{\jmath-k}^{L}$ & $-q_{j-k}^{L, g a s}$ & $q_{f-k}^{\prime \prime f i r e} \alpha_{f-k}^{L}$ \\
\hline $\begin{array}{l}\text { from the upper to } \\
\text { the lower layer }\end{array}$ & $q_{j-k}^{\text {out }} \tau_{j-k}^{U} \alpha_{j-k}^{L}$ & $q_{j-k}^{U, g a s} \alpha_{j-k}^{L}-q_{j-k}^{L, g a s}$ & $q_{f-k}^{\prime \prime f i r e} \alpha_{f-k}^{L} \tau_{f-k}^{U}$ \\
\hline $\begin{array}{l}\text { from the upper to } \\
\text { the upper layer }\end{array}$ & 0 & 0 & 0 \\
\hline
\end{tabular}

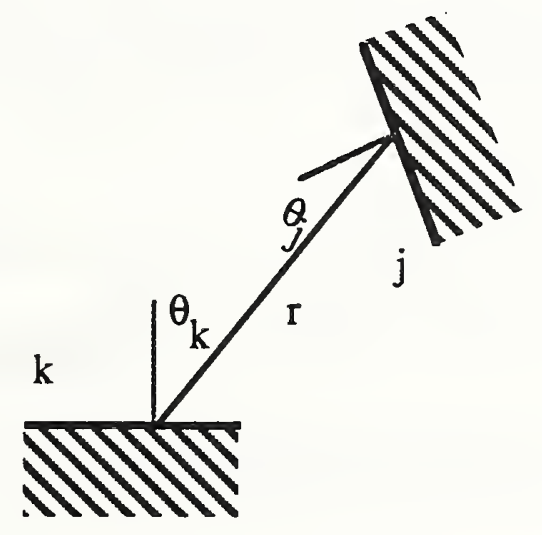

Figure 5: Configuration Setup 


$$
F_{j-k}=\frac{1}{A_{j}} \iint_{A_{j} A_{k}} \frac{\cos \left(\theta_{j}\right) \cos \left(\theta_{k}\right)}{\pi r^{2}} d A_{k} d A_{j} .
$$

The following properties can be derived from this definition for any wall segments

$$
\begin{aligned}
A_{j} F_{j-k} & =A_{k} F_{k-j} \\
F_{i-j \oplus k} & =F_{i-j}+F_{i-k} \\
A_{i \oplus j} F_{i \oplus j-k} & =A_{i} F_{i-k}+A_{j} F_{j-k}
\end{aligned}
$$

where $i \oplus j$ denotes the union of two wall surface $\mathrm{i}$ and $\mathrm{j}$.

If the collection of $\mathrm{N}$ wall segments form an enclosure (i.e. a room) then the configuration factors also satisfy equation (10). This occurs since the energy leaving the $\mathrm{j}$ 'th surface must strike one of the $\mathrm{N}$ surfaces in the enclosure (possibly itself).

$$
\sum_{k=1}^{N} F_{j-k}=1 j=1, \ldots, N
$$

If four wall segments $1 \ldots 4$ are configured as illustrated in Figure 6 then the following configuration factor identity also holds.

$$
A_{1} F_{1-4}=A_{2} F_{2-3}
$$

By assuming that two wall segments are either parallel or perpendicular then only two classes of configuration factors need to be calculated directly in a zone fire model radiation algorithm. Equation (6) is used to derive configuration factors for simple geometries that occur in zone fire modeling. A catalog of configuration factor formulas for various geometric configurations is given in [7, Appendix C]. Direct configuration factor formulas used in RAD2, RAD4 and RAD10 follow.

Consider two finite rectangles perpendicular to each other having a common edge of the same length as illustrated in Figure 7. The dimensions of the "from" rectangle is $l \times w$ and the dimension of the "to" rectangle is $l \times h ; l$ is common to both rectangles. The configuration factor, $\phi_{\text {perp }}(h, l, w)$, from rectangle 1 to 2 is then

$$
\begin{aligned}
F_{1-2} & =\phi_{\text {perp }}(h, l, w)= \\
& \frac{1}{\pi W}\left\{W \operatorname{Tan}^{-1} \frac{1}{W}+H \operatorname{Tan}^{-1} \frac{1}{H}-\sqrt{H^{2}+W^{2}} \operatorname{Tan}^{-1} \frac{1}{\sqrt{H^{2}+W^{2}}}+\right. \\
& \frac{1}{4} \log \left\{\frac{\left(1+W^{2}\right)\left(1+H^{2}\right)}{1+H^{2}+W^{2}}\left[\frac{W^{2}\left(1+H^{2}+W^{2}\right)}{\left(1+W^{2}\right)\left(H^{2}+W^{2}\right)}\right]^{W^{2}}\right. \\
& \left.\left.\times\left[\frac{H^{2}\left(1+H^{2}+W^{2}\right)}{\left(1+W^{2}\right)\left(H^{2}+W^{2}\right)}\right]^{H^{2}}\right\}\right\}
\end{aligned}
$$

where $H=h / l$ and $W=w / l$. This formula is found in [7, p. 825].

We also need to compute the configuration factor between two identical, parallel, directly opposed rectangles. This situation is setup in Figure 7 . The distance between the two 


$$
A_{1} F_{1-4}=A_{2} F_{2-3}
$$
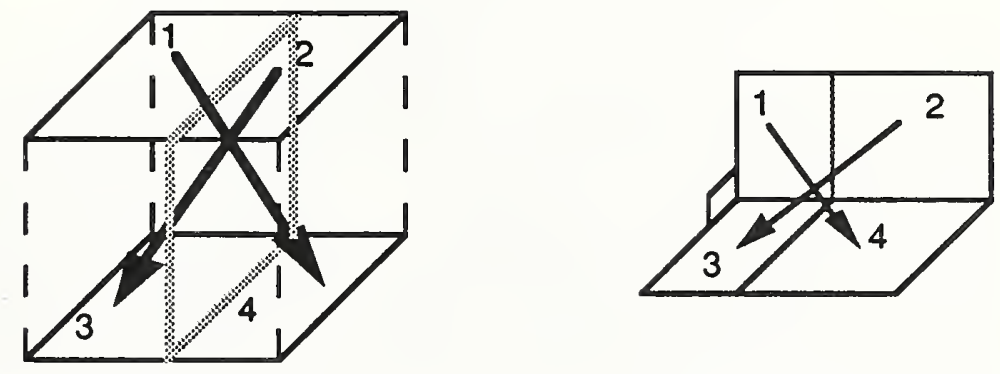

Figure 6: Configuration Factor Symmetries
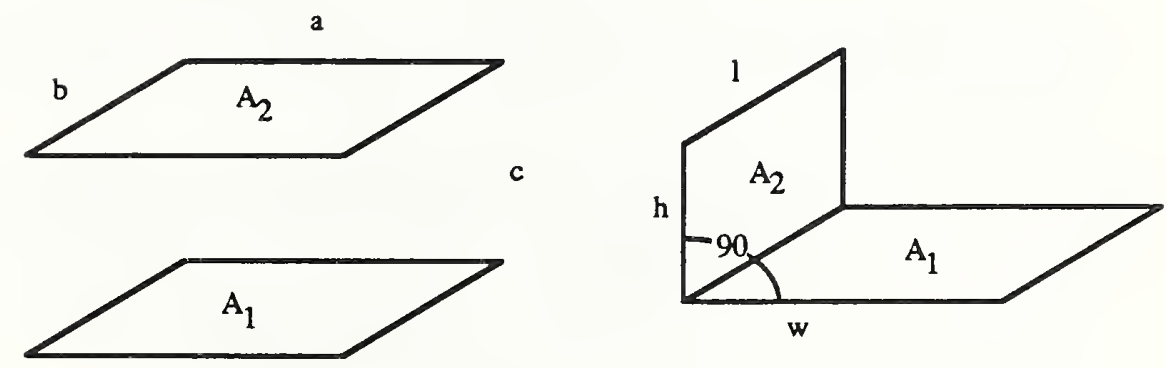

Figure 7: Configuration Factor Setup For Pairs of Parallel and Perpendicular Rectangles 
rectangles is $c$. The length and width of these rectangles is $a$ and $b$. The configuration factor, $\phi_{p a r}(a, b, c)$ between these two rectangles is then

$$
\begin{aligned}
& F_{1-2}=\phi_{p a r}(a, b, c)= \\
& \quad \frac{1}{\pi X Y}\left\{\log \sqrt{\frac{\left(1+X^{2}\right)\left(1+Y^{2}\right)}{1+X^{2}+Y^{2}}}+X \sqrt{1+Y^{2}} \operatorname{Tan}^{-1} \frac{X}{1+Y^{2}}+\right. \\
& \left.Y \sqrt{1+X^{2}} \operatorname{Tan}^{-1} \frac{Y}{1+X^{2}}\right\} .
\end{aligned}
$$

where $X=a / c$ and $Y=b / c$. This formula is symmetric in $X$ and $Y$. It can be found in [7, p. 824].

For a room with $\mathrm{N}$ wall segments, $N \times N=N^{2}$ configuration factors must be calculated. Equations (12) and (13) are expensive to compute due to the complicated expressions involving $\log$ and $\operatorname{Tan}^{-1}$ functions. This portion of the work is reduced in RAD4 by noting that only 2 configuration factor calculations involving equation (12) are required rather than $4 \times 4=16$. The other 14 configuration factors are obtained using algebraic relationships. These algebraic formulas are given in section 2.2.2. For the RAD10 case only eight configuration factor calculations need be calculated using equations (12) and (13). Again, the other 92 can be obtained using algebraic formulas. These formulas are detailed in section 2.2 .3 .

\subsubsection{Solid Angles}

A solid angle as illustrated in figure 8 is the area intercepted on a unit sphere by a conical angle originating at the sphere center. A solid angle is used to determine the fraction of a radiating point source fire that strikes a surface. This formula was obtained from Yamada's formula in [10] by observing that

$$
\operatorname{Sin}^{-1}\left(\frac{x}{\sqrt{x^{2}+y^{2}}}\right)+\operatorname{Sin}^{-1}\left(\frac{y}{\sqrt{x^{2}+y^{2}}}\right)=\frac{\pi}{2} .
$$

The solid angle of a rectangle with sides of length $x$ and $y$ that lies in a plane a distance $r$ from the center of a sphere is given by

$$
\omega(x, y)=\frac{1}{4 \pi}\left\{\operatorname{Sin}^{-1}\left(A \frac{y}{\sqrt{y^{2}+r^{2}}}\right)+\operatorname{Sin}^{-1}\left(A \frac{x}{\sqrt{x^{2}+r^{2}}}\right)-\frac{\pi}{2}\right\}
$$

where

$$
A=\sqrt{1+\frac{r^{2}}{x^{2}+y^{2}}}
$$

The solid angle $\omega(x, y)$ is symmetric in $x$ and $y$. Solid angles are also additive, so that the solid angle of an arbitrary rectangle can be computed using (14) and

$$
\begin{aligned}
\omega\left(x_{1}, x_{2}, y_{1}, y_{2}\right)= & \operatorname{sgn}\left(x_{2} y_{2}\right) \omega\left(\left|x_{2}\right|,\left|y_{2}\right|\right)-\operatorname{sgn}\left(x_{2} y_{1}\right) \omega\left(\left|x_{2}\right|,\left|y_{1}\right|\right)- \\
& \operatorname{sgn}\left(x_{1} y_{2}\right) \omega\left(\left|x_{1}\right|,\left|y_{2}\right|\right)+\operatorname{sgn}\left(x_{1} y_{1}\right) \omega\left(\left|x_{1}\right|,\left|y_{1}\right|\right)
\end{aligned}
$$




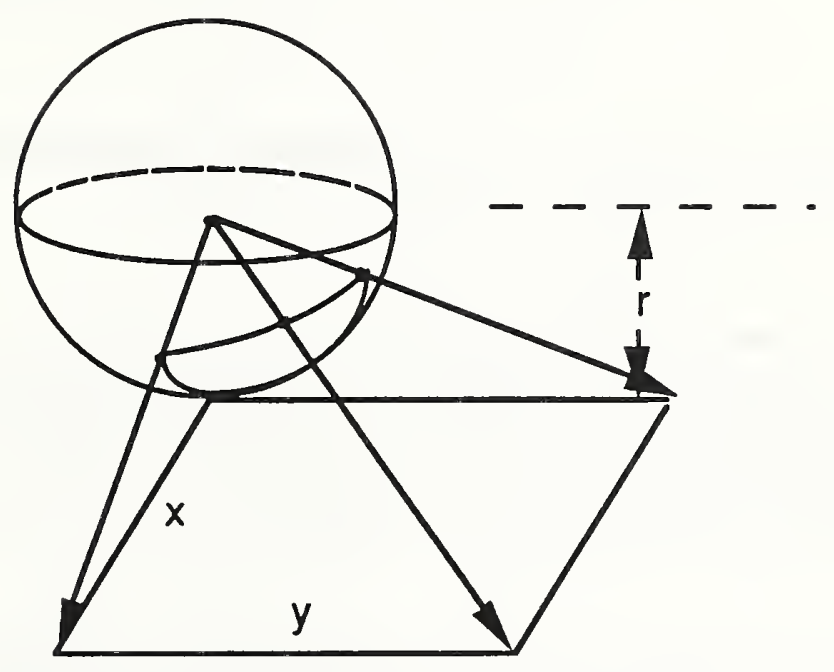

Figure 8: Solid Angle Setup

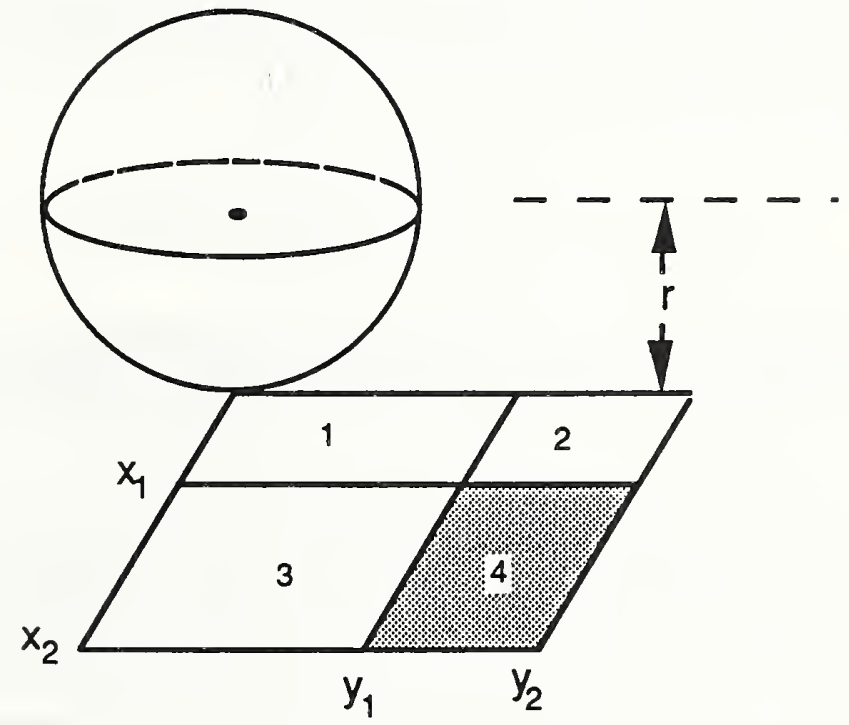

$\omega_{\mathrm{i}}=$ solid angle for rectangle $\mathrm{i}$

$\omega_{4}=\omega_{1+2+3+4}-\omega_{1+2}-\omega_{1+3}+\omega_{1}$

Figure 9: Solid Angles For Arbitrary Rectangles 


$$
\operatorname{sgn}(x)= \begin{cases}1 & \text { if } x \geq 0 \\ -1 & \text { if } x<0\end{cases}
$$

Figure 9 illustrates how the solid angle of rectangle 4 can be computed in terms of solid angles of rectangles $1 \oplus 2 \oplus 3 \oplus 4,1 \oplus 3,1 \oplus 2$ and 1 .

\subsubsection{Transmission Factors}

A transmission factor, $\tau$, is the fraction of energy passing through a gas unimpeded. The transmittance of a gas depends on the absorption coefficient of the gas and the length of the path through the gas. A simple relationship for $\tau$ can be determined by assuming that the absorptance of the gas (a local phenomena) is uniform throughout the gas layer. This factor, a decaying exponential, is given by

$$
\tau(L)=e^{-A L}
$$

where $\mathrm{A}$ is the absorptance of the gas per unit length and $\mathrm{L}$ is the path length. This formula is known as Beer's law. The gas absorptance is not calculated by the radiation exchange algorithms presented in this report. Modak in [14] gives an algorithm for calculating gas absorptance from such information as soot concentration, partial pressures of $\mathrm{CO}, \mathrm{CO} 2$ etc. The emittance of the gas is the same as its absorption due to the gray gas assumption. The transmission factor, $\tau$ in the above equation is defined for one specific path through a gas. We are, however considering radiation exchange between a pair of finite area rectangles where many paths of different lengths occur. Siegel and Howell define an average transmission factor [7, p. 603] considering all possible paths between two surfaces through the gas. This form of $\tau$ is defined to be

$$
\overline{\tau_{j-k}}=\iint_{A, A_{k}} \frac{\tau(L) \cos \left(\theta_{k}\right) \cos \left(\theta_{j}\right)}{\pi L^{2}} d A_{j} d A_{k} /\left(A_{j} F_{j-k}\right)
$$

The numerator of the fraction in in the above equation is the same as a configuration factor if $\tau(L)=1$. It is not practical to compute a transmission factor using this integral. We can estimate this integral by finding a characteristic path with length $\bar{L}$ such that

$$
\overline{\tau_{j-k}}=e^{-A \bar{L}} \text {. }
$$

For the ten-wall model, this path is taken to be between the centers of two rectangles. This length is an underestimate of $\bar{L}$. This approximation breaks down when the two wall segments are close together or one of the wall segments is a complex shape (such as the union of four upper walls). The four and two-wall model estimates $\bar{L}$ based upon an average distance between the rectangles that make up the wall segments.

For a given path between surface $\mathrm{j}$ and surface $\mathrm{k}$ we need to calculate the path length through the upper layer, $L_{U}$, and the path length through the lower layer $L_{L}$. Transmission factors for the upper and lower layers are then defined to be

$$
\begin{aligned}
\tau_{j-k}^{U} & =e^{-L_{j-k}^{U} A_{U}}, \\
\tau_{j-k}^{L} & =e^{-L_{j-k}^{L} A_{L}} .
\end{aligned}
$$


The fraction of energy that passes through both layers is then

$$
\tau_{j-k}=\tau_{j-k}^{U} \tau_{j-k}^{L} .
$$

The amount of energy absorbed by a layer is just the amount that doesn't pass through a layer or

$$
\begin{aligned}
& \alpha_{j-k}^{U}=1-\tau_{j-k}^{U}=1-e^{-L_{j-k}^{U} A_{U}}, \\
& \alpha_{j-k}^{L}=1-\tau_{j-k}^{L}=1-e^{-L_{j-k}^{L} A_{L}} .
\end{aligned}
$$

\subsection{Two, Four, Ten Wall Segment Radiation Exchange}

The formulas in section 2.1 for computing radiation exchange were specified in terms of general wall segments. This section discusses the radiation exchange computation in terms of a two-wall, four-wall and ten-wall model.

\subsubsection{Two-Wall Configuration Factors}

The two-wall model combines the ceiling and four upper walls into one wall segment and the four lower walls and the floor into the second wall segment. The configuration factors for these two surfaces are derived by Quintiere in [15, Appendix] and are

$$
\begin{aligned}
& F_{1-1}=1-\frac{A_{D}}{A_{1}}, \\
& F_{1-2}=\frac{A_{D}}{A_{1}}, \\
& F_{2-1}=\frac{A_{D}}{A_{2}}, \\
& F_{2-2}=1-\frac{A_{D}}{A_{2}}
\end{aligned}
$$

where $A_{1}, A_{D}$ and $A_{2}$ are the areas of the extended ceiling, layer interface and extended floor respectively. These configuration factors are used in the original two-wall radiation model in CFAST [5] and in BRI [3, 4].

The two-wall model, RAD2, interacts with a four-wall heat conduction model in CFAST. The ceiling and upper wall temperatures may be different, so the question of how to represent the extended ceiling temperature arises. RAD2 chooses an extended ceiling temperature that results in the same energy contribution to the enclosure that a four-wall radiation algorithm would predict. The energy added to the room due to the ceiling and upper wall temperatures of $T_{1 a}$ and $T_{1 b}$ is

$$
\sigma\left(A_{1 a} \epsilon_{1 a} T_{1 a}^{4}+A_{1 b} \epsilon_{1 b} T_{1 b}^{4}\right) .
$$

We want to choose an effective or average temperature, $T_{a v g}$, and emittance, $\epsilon_{a v g}$ for the extended ceiling that matches this energy contribution.

$$
\overbrace{\sigma\left(A_{1 a}+A_{1 b}\right) \epsilon_{a v g} T_{a v g}^{4}}^{\text {energy from extended ceiling }}=\overbrace{\sigma A_{1 a} \epsilon_{1 a} T_{1 a}^{4}}^{\text {energy from ceiling }}+\overbrace{\sigma A_{1 b} \epsilon_{1 b} T_{1 b}^{4}}^{\text {energy from upper wall }}
$$


An average emittance for the two wall segments is computed using an average of $\epsilon_{1 a}$ and $\epsilon_{1 b}$ weighted by wall segment areas or

$$
\epsilon_{a v g}=\frac{A_{1 a} \epsilon_{1 a}+A_{1 b} \epsilon_{1 b}}{A_{1 a}+A_{1 b}} .
$$

Equation (15) can now be solved for $T_{a v g}$ using this value of $\epsilon_{a v g}$ to obtain

$$
T_{a v g}=\sqrt[4]{\frac{A_{1 a} \epsilon_{1 a} T_{1 a}^{4}+A_{1 b} \epsilon_{1 b} T_{1 b}^{4}}{A_{1 a} \epsilon_{1 a}+A_{1 b} \epsilon_{1 b}}} .
$$

A similar procedure can be used to compute an effective temperature and emittance for the extended floor.

\subsubsection{Four-Wall Configuration Factors}

The configuration factors for four-wall radiation exchange are derived similarly to Quintiere's derivation for two walls in [15, Appendix]. The setup for the following derivation is given in Figure 11. We wish to determine the configuration factors

$$
F_{i-j} \text { for } i, j=1, \ldots, 4 \text {. }
$$

The 16 configuration factors can be determined in terms of $F_{1-4}, F_{1-d}$ and $F_{4-d} . F_{1-4}$ does not change during a simulation since its value depends only on the height of the room and the area of the floor. Therefore, $F_{1-4}$ only needs to be computed once. Configuration factors, $F_{1-d}$ and $F_{4-d}$ depend on the layer interface height so need to be calculated each time the radiation exchange is to be calculated. Configuration factors $F_{1-4}, F_{1-d}$ and $F_{4-d}$ are determined using equation (13). Since $A_{1}=A_{4}$ it follows that $F_{4-1}=F_{1-4}$. The other 14 configuration factors can be calculated using simple algebraic formulas.

Since the floor and the ceiling is assumed to be a flat rectangular surface it follows that

$$
F_{1-1}=F_{4-4}=0 \text {. }
$$

Using the fact that configuration factors in an enclosure sum to 1 and that due to symmetry $F_{2-1}=F_{2-d}$, it follows that

$$
\begin{array}{rcc}
F_{1-2}+F_{1-d} & = & 1 \\
F_{2-1}+F_{2-2}+F_{2-d} & =2 F_{2-1}+F_{2-2}= & 2 \frac{A_{1}}{A_{2}} F_{1-2}+F_{2-2}=1
\end{array}
$$

Equations (16) and (17) can be solved for $F_{1-2}$ and $F_{2-2}$ to obtain

$$
\begin{aligned}
& F_{1-2}=1-F_{1-d} \\
& F_{2-1}=\frac{A_{1}}{A_{2}} F_{1-2} \\
& F_{2-2}=1-2 F_{2-1}
\end{aligned}
$$

Similarly,

$$
\begin{aligned}
F_{4-3} & =1-F_{4-d} \\
F_{3-4} & =\frac{A_{4}}{A_{3}} F_{4-3} \\
F_{3-3} & =1-2 F_{3-4}
\end{aligned}
$$




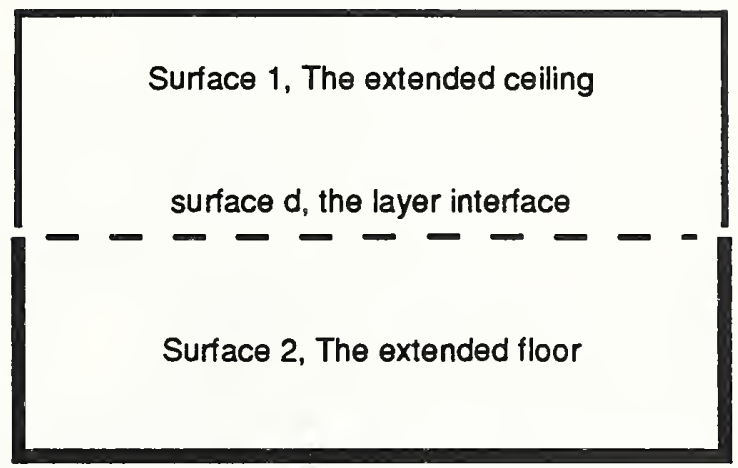

Figure 10: Extended Floor, Ceiling Configuration Factor Setup

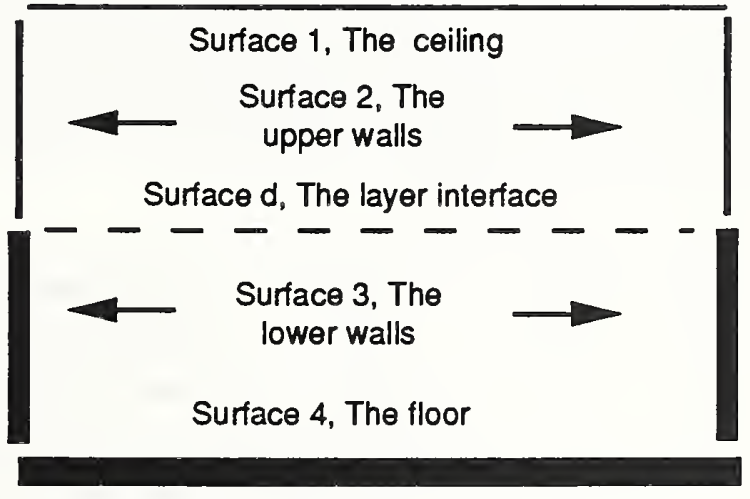

Figure 11: Four Wall Configuration Factor Setup 
Using the above configuration factors and equation (10) it follows that

$$
\begin{aligned}
& F_{1-3}=1-F_{1-4}-F_{1-2} \\
& F_{3-1}=\frac{A_{1}}{A_{3}} F_{1-3} \\
& F_{3-2}=1-F_{3-1}-F_{3-3}-F_{3-4} \\
& F_{2-3}=\frac{A_{3}}{A_{2}} F_{3-2} \\
& F_{2-4}=1-F_{2-1}-F_{2-2}-F_{2-3} \\
& F_{4-2}=\frac{A_{2}}{A_{4}} F_{2-4}
\end{aligned}
$$

\subsubsection{Ten-Wall Configuration Factors}

To handle the more general radiation exchange case, a room is split into ten surfaces as illustrated in Figure 12. These surfaces are the ceiling, four upper walls, four lower walls and the floor. The radiation exchange is computed between these ten surfaces and the intervening gas layer(s). In general, 100 configuration factors, $F_{j-k}$ and 100 transmission factors $\tau_{j-k}$ need to be determined each time this algorithm is invoked. Although there are 100 configuration factors for this room, only eight have to be calculated directly using equations (12) and (13). The other 92 can be computed in terms of simple algebraic relationships using the properties outlined in equations (7) to (11). This reduction in required configuration factor calculations is due to the fact that the rectangle pairs 2 and 4,3 and 5,6 and 8 and 7 and 9 each have equal areas.

The following 15 configuration factors are computed only once during a fire simulation, assuming that the room does not change size. These factors are between the six surfaces that form the enclosure. There are a total of 36 configuration factors between these six surfaces. The six factors, $F_{k-k}$, are each zero. Fifteen factors are given below. The other 15 can be derived using the symmetry relation $A_{j} F_{j-k}=A_{k} F_{k-j}$. The first six configuration factors are computed using formulas (12), (13). The other nine configuration factors are derived from these six using the identities (7) through (11). These formulas are implemented in the FORTRAN subroutine RMFIG

$$
\begin{aligned}
F_{1-2 \oplus 6} & =\phi_{\text {perp }}(\Delta z, \Delta x, \Delta y) \\
F_{1-3 \oplus 7} & =\phi_{\text {perp }}(\Delta z, \Delta y, \Delta x) \\
F_{1-10} & =\phi_{\text {par }}(\Delta x, \Delta y, \Delta z) \\
F_{2 \oplus 6-3 \oplus 7} & =\phi_{\text {perp }}(\Delta y, \Delta z, \Delta x) \\
F_{2 \oplus 6-, 4 \oplus 8} & =\phi_{\text {par }}(\Delta x, \Delta z, \Delta y) \\
F_{3 \oplus 7-5 \oplus 9} & =\phi_{\text {par }}(\Delta z, \Delta y, \Delta x)
\end{aligned}
$$

where $\phi_{\text {perp }}$ is the configuration factor for two perpendicular rectangles sharing a common edge given by equation (12) and $\phi_{p a r}$ is the configuration factor for two identical parallel rectangles given by equation (13).

$$
F_{1-4 \oplus 8}=F_{1-2 \oplus 6}
$$




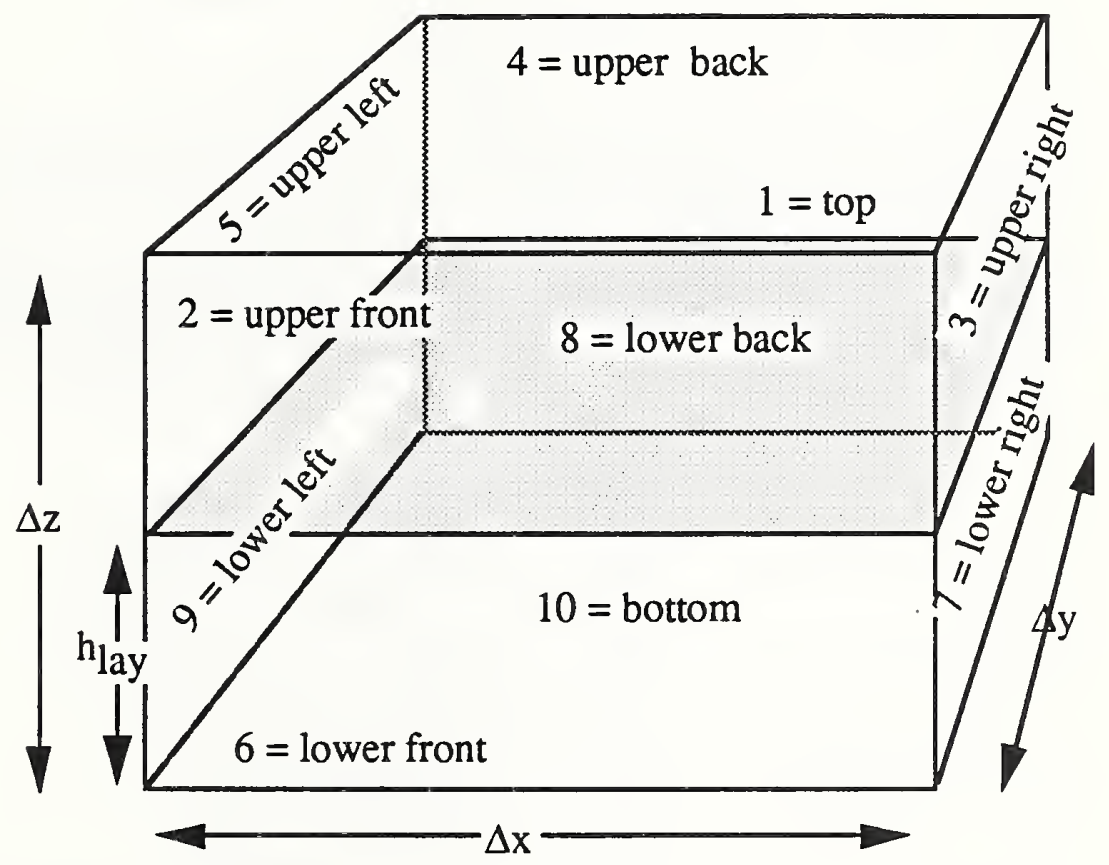

Figure 12: Ten Wall Configuration Factor Setup 


$$
\begin{aligned}
F_{1-5 \oplus 9} & =F_{1-3 \oplus 7} \\
F_{2 \oplus 6-5 \oplus 9} & =F_{2 \oplus 6-3 \oplus 7} \\
F_{2 \oplus 6-10} & =F_{2 \oplus 6-1} \\
F_{3 \oplus 7-4 \oplus 8} & =F_{3 \oplus 7-2 \oplus 6} \\
F_{3 \oplus 7-10} & =F_{3 \oplus 7-1} \\
F_{4 \oplus 8-5 \oplus 9} & =F_{4 \oplus 8-3 \oplus 7} \\
F_{4 \oplus 8-10} & =F_{4 \oplus 8-1} \\
F_{5 \oplus 9-10} & =F_{5 \oplus 9-1}
\end{aligned}
$$

The following configuration factors are computed each time the radiation exchange is computed since these terms depend on the layer height which may vary. The first eight configuration factors are computed using formulas (12), (13). The other 92 configuration factors are derived from these eight using the identities (7) to (11). The formulas are listed in the order that they are computed so that once a configuration factor appears on the left hand side of an equation (such as $F_{1-6}$ ) it may be used later on the right hand side. These formulas are implemented in the FORTRAN subroutine LAYFIG.

$$
\begin{aligned}
& F_{1-2}=\phi_{\text {perp }}(\Delta z-h l a y, \Delta x, \Delta z) \\
& F_{1-3}=\phi_{\text {perp }}(\Delta z-h l a y, \Delta y, \Delta x) \\
& F_{2-3}=\phi_{\text {perp }}(\Delta y, \Delta z-h l a y, \Delta x) \\
& F_{2-4}=\phi_{\text {par }}(\Delta x, \Delta z-h l a y, \Delta y) \\
& F_{3-5}=\phi_{\text {par }}(\Delta z-\text { hlay, } \Delta y, \Delta x) \\
& F_{6-7}=\phi_{\text {perp }}(\Delta y, \text { hlay, } \Delta x) \\
& F_{6-8}=\phi_{\text {par }}(h l a y, \Delta x, \Delta y) \\
& F_{7-9}=\phi_{\text {par }}(\Delta y, h l a y, \Delta x)
\end{aligned}
$$

First row and column

$$
\begin{aligned}
F_{1-1} & =0 \\
F_{1-4} & =F_{1-2} \\
F_{1-5} & =F_{1-3} \\
F_{1-6} & =F_{1-2 \oplus 6}-F_{1-2} \\
F_{1-7} & =F_{1-2 \oplus 7}-F_{1-3} \\
F_{1-8} & =F_{1-6} \\
F_{1-9} & =F_{1-7} \\
F_{1-10} & =\phi_{p a r}(\Delta x, \Delta y, \Delta z) \quad \text { (computed only once) } \\
F_{j-1} & =\frac{A_{1}}{A_{j}} F_{1-j} j=2, \ldots, 10
\end{aligned}
$$




\section{Second Row and Column}

$$
\begin{aligned}
& F_{2-2}=0 \\
& F_{2-5}=F_{2-3} \\
& F_{2-6}=0
\end{aligned}
$$

The formula for $F_{2-7}$ will be derived. Derivations for other factors follow similarly. Equations (8) and (9) can be used to simplify $A_{2 \oplus 6} F_{2 \oplus 6-3 \oplus 7}$ to

$$
\begin{aligned}
A_{2 \oplus 6} F_{2 \oplus 6-3 \oplus 7} & =A_{2} F_{2-3 \oplus 7}+A_{6} F_{6-3 \oplus 7} \\
& =A_{2} F_{2-3}+A_{2} F_{2-7}+A_{6} F_{6-3}+A_{6} F_{6-7} .
\end{aligned}
$$

The factors $F_{2-7}$ and $F_{6-3}$ are related via $A_{2} F_{2-7}=A_{6} F_{6-3}$ since the surfaces $2,3,6$ and 7 are configured as illustrated in figure 6 . Equation (18) can then be simplified to

$$
A_{2 \oplus 6} F_{2 \oplus 6-3 \oplus 7}=A_{2} F_{2-3}+2 A_{2} F_{2-7}+A_{6} F_{6-7}
$$

Solving for $F_{2-7}$ yields the desired formula as listed below. Note that $F_{2 \oplus 3-3 \oplus 7}, F_{2-3}$ and $F_{6-7}$ were computed previously.

$$
\begin{aligned}
F_{2-7} & =\frac{A_{2 \oplus 6} F_{2 \oplus 6-3 \oplus 7}-A_{2} F_{2-3}-A_{6} F_{6-7}}{2 A_{2}} \\
F_{2-8} & =\frac{A_{2 \oplus 6} F_{2 \oplus 6-4 \oplus 8}-A_{2} F_{2-4}-A_{6} F_{6-8}}{2 A_{2}} \\
F_{2-9} & =F_{2-7} \\
F_{2-10} & =1-\sum_{j=1}^{9} F_{2-j} \\
F_{j-2} & =\frac{A_{2}}{A_{j}} F_{2-j} j=3, \ldots, 10
\end{aligned}
$$

\section{Third Row and Column}

$$
\begin{aligned}
F_{3-3} & =0 \\
F_{3-4} & =F_{3-2} \\
F_{3-6} & =\frac{A_{2}}{A_{3}} F_{2-7} \\
F_{3-7} & =0 \\
F_{3-8} & =F_{3-6} \\
F_{3-9} & =\frac{A_{3 \oplus 7} F_{3 \oplus 7-5 \oplus 9}-A_{3} F_{3-5}-A_{7} F_{7-9}}{2 A_{3}} \\
F_{3-10} & =1-\sum_{j=1}^{9} F_{3-j} \\
F_{j-3} & =\frac{A_{3}}{A_{j}} F_{3-j} j=4, \ldots, 10
\end{aligned}
$$


Fourth Row and Column

$$
\begin{aligned}
F_{4-4} & =0 \\
F_{4-5} & =F_{2-3} \\
F_{4-6} & =F_{2-8} \\
F_{4-7} & =F_{2-7} \\
F_{4-8} & =0 \\
F_{4-9} & =F_{2-9} \\
F_{4-10} & =F_{2-10} \\
F_{j-4} & =\frac{A_{4}}{A_{j}} F_{4-j} j=5, \ldots, 10
\end{aligned}
$$

Fifth Row and Column

$$
\begin{aligned}
F_{5-5} & =0 \\
F_{5-6} & =F_{3-6} \\
F_{5-7} & =F_{3-9} \\
F_{5-8} & =F_{3-8} \\
F_{5-9} & =0 \\
F_{5-10} & =F_{3-10} \\
F_{j-5} & =\frac{A_{5}}{A_{j}} F_{5-j} j=6, \ldots, 10
\end{aligned}
$$

Sixth Row and Column

$$
\begin{aligned}
F_{6-6} & =0 \\
F_{6-9} & =F_{6-7} \\
F_{6-10} & =\frac{A_{2 \oplus 6} F_{2 \oplus 6-10}-A_{2} F_{2-10}}{A_{6}} \\
F_{j-6} & =\frac{A_{6}}{A_{j}} F_{6-j} j=7, \ldots, 10
\end{aligned}
$$

Seventh Row and Column

$$
\begin{aligned}
F_{7-7} & =0 \\
F_{7-8} & =F_{7-6} \\
F_{7-10} & =\frac{A_{3 \oplus 7} F_{3 \oplus 7-10}-A_{3} F_{3-10}}{A_{7}} \\
F_{j-7} & =\frac{A_{7}}{A_{j}} F_{7-j} j=8, \ldots, 10
\end{aligned}
$$

Eight Row and Column 


$$
\begin{aligned}
F_{8-8} & =0 \\
F_{8-9} & =F_{6-9} \\
F_{8-10} & =F_{6-10} \\
F_{j-8} & =\frac{A_{8}}{A_{j}} F_{8-j} j=9, \ldots, 10
\end{aligned}
$$

Ninth Row and Column

$$
\begin{aligned}
F_{9-9} & =0 \\
F_{9-10} & =F_{7-10} \\
F_{10-9} & =\frac{A_{9}}{A_{10}} F_{9-10}
\end{aligned}
$$

Tenth Row and Column

$$
F_{10-10}=0
$$

\section{Solving the Net Radiation Equations}

\subsection{Solving The Net Radiation Equations Efficiently}

The net radiation equation (5) is not diagonally dominant. Iterative methods then should not be used to solve this equation unless it is suitably transformed. This can be done by substituting, $\Delta q^{\prime \prime}{ }_{k}=\epsilon_{k} \Delta \hat{q}_{k}^{\prime \prime}$ into equation (5) to obtain

$$
\Delta \hat{q}_{k}^{\prime \prime}-\sum_{j=1}^{N}\left(1-\epsilon_{j}\right) \Delta \hat{q}_{j}^{\prime \prime} F_{k-j} \tau_{j-k}=\sigma T_{k}^{4}-\sum_{j=1}^{N} \sigma T_{j}^{4} F_{k-j} \tau_{j-k}-\frac{c_{k}}{A_{k}} .
$$

There are two reasons for solving equation (19) instead of (5). First, since $\epsilon_{k}$ does not occur in the denominator, radiation exchange can be calculated when a wall segment emittance is zero. Second and more importantly, the matrix corresponding to the linear system of equations in (19) is diagonally dominant. When the number of wall segments is large and the wall segments have emittances close to one which often occurs in typical fire scenarios, the time required to solve this modified linear system can be significantly reduced due to this diagonal dominance by using iterative methods.

To see this, re-write equation (5) into matrix form to obtain

$$
A \Delta q^{\prime \prime}=B E-c
$$

where the $k, j$ 'th components of the $N \times N$ matrices $A$ and $B$ are

$$
\begin{aligned}
a_{k, j} & =\frac{\delta_{k, j}}{\epsilon_{j}}-F_{k-j} \tau_{j-k} \frac{1-\epsilon_{j}}{\epsilon_{j}} \\
b_{k, j} & =\delta_{k, j}-F_{k-j} \tau_{j-k}
\end{aligned}
$$


and the k'th component of the vectors $c^{\prime \prime}$ and $E$ are

$$
\begin{aligned}
c_{k}^{\prime \prime}=\frac{c_{k}}{A_{k}} & =\sum_{j=1}^{N}\left(\begin{array}{c}
q_{j-k}^{\prime U, g a s}+q_{j-k}^{\prime L, g a s} \\
j-k
\end{array}\right)+\sum_{j=1}^{N_{f i r e}} q_{\substack{\prime f i r e \\
f-k}}^{f^{\prime}}, \\
E_{k} & =\sigma T_{k}^{4},
\end{aligned}
$$

and $\delta_{k, j}$ is the dirac delta function, $F_{k-j}$ is the configuration factor from the k'th to the j'th wall segment, $\epsilon_{j}$ is the emittance of the $j$ 'th wall segment, $\tau_{j-k}$ is a fraction ranging from 0 to 1 indicating the amount of radiation that is transmitted through a gas. Also, $q_{j-k}^{\prime \prime U}$,gas and $q_{j-k}^{\prime \prime L, g a s}$ are radiation contributions due to the gas layers and $q_{j-k}^{\prime \prime f i r e}$ are radiation terms due to the f'th fire. The matrix $A$ can be transformed into a diagonally dominant matrix using the following scaling matrix,

$$
D=\left(\begin{array}{ccc}
\varepsilon_{1} & 0 & 0 \\
0 & \ddots & 0 \\
0 & 0 & \varepsilon_{N}
\end{array}\right)
$$

where $\epsilon_{k}$ is the emittance of the k'th wall segment. Define the scaled matrix $\hat{A}$ by postmultiplying $\mathrm{A}$ by $\mathrm{D}$ and pre-multiplying $\Delta q^{\prime \prime}$ by $D^{-1}$ to obtain

$$
\begin{aligned}
\hat{A} & =A D \\
\Delta \hat{q}^{\prime \prime} & =D^{-1} \Delta q^{\prime \prime} .
\end{aligned}
$$

Equation (20) then reduces to

$$
\hat{A} \Delta \hat{q}^{\prime \prime}=A \Delta q^{\prime \prime}=B E-c .
$$

Once the solution $\Delta \hat{q}^{\prime \prime}$ is found we may recover the solution, $\Delta q^{\prime \prime}$, to the original problem by using $\Delta q^{\prime \prime}=D \Delta \hat{q}^{\prime \prime}$. The matrix $\hat{A}$ is diagonally dominant which is now shown.

Using the definition of $a_{k, j}$ in equation (21) the kj'th element of $\hat{A}$ is

$$
\hat{a}_{k, j}=a_{k, j} \epsilon_{j}=\delta_{k, j}-F_{k-j} \tau_{j-k}\left(1-\epsilon_{j}\right) .
$$

A matrix is diagonally dominant if for each row the absolute value of the diagonal element is greater than the sum of the absolute values of the off diagonal elements or equivalently

$$
\left|\hat{a}_{k, k}\right|>\sum_{\substack{j=1 \\ j \neq k}}^{N}\left|\hat{a}_{k, j}\right|
$$

Substituting (25) into (26) we get the following requirement for $\hat{A}$ to be diagonally dominant.

$$
1-F_{k-k}\left(1-\varepsilon_{k}\right) \tau_{k-k}>\sum_{\substack{j=1 \\ j \neq k}}^{N} F_{k-j}\left(1-\varepsilon_{j}\right) \tau_{j-k}
$$


or equivalently

$$
1>\sum_{j=1}^{N} F_{k-j}\left(1-\epsilon_{j}\right) \tau_{j-k} .
$$

The matrix $\hat{A}$ is then diagonally dominant since $1>\left(1-\epsilon_{j}\right) \tau_{j-k}$ and

$$
1=\sum_{j=1}^{N} F_{k-j}>\sum_{j=1}^{N} F_{k-j}\left(1-\varepsilon_{j}\right) \tau_{j-k} .
$$

Iterative techniques ${ }^{6}$ are guaranteed to converge for diagonally dominant matrices [16, p. 542]. They also can be much more efficient than direct methods such as Gaussian elimination. The convergence speed depends on how small the right hand side of the above inequality is compared to 1 . Physically, if the surfaces being modeled are approximate black bodies ( $\epsilon$ close 1$)$ or the gas layers are thick ( $\tau$ close to 0$)$ then iterative techniques for solving the net radiation equations will converge rapidly. Typical emittances for materials used in fire simulations range from $\epsilon=.85$ to .95 . For the limiting case when the wall materials are black bodies then the matrix $\hat{A}$ is a diagonal matrix and iterative methods will converge in one iteration.

The advantage of using an iterative method over a direct method for computing radiation exchange between approximate black bodies increases as the number of wall segments increases. The cost of solving the linear system directly is proportional $\frac{2}{3} N^{3}$ while the cost of using iterative techniques is proportional to $k N^{2}$ where $\mathrm{k}$ is the number of iterations and $\mathrm{N}$ is the number of wall segments. Using Gauss-Seidel iterative methods, it has been found that convergence is achieved after two to three iterations for emittances around .9. The break-even point between iterative and direct methods for matrices of size 10 is about 6 or 7 . The linear system for RAD2 and RAD4 is $2 \times 2$ and $4 \times 4$ respectively. Iterative methods are not faster for problems this small. RAD10 and problems with more wall segments can use iterative methods to decrease the time required to solve the linear system without sacrificing accuracy.

The radiation exchange equations can be solved analytically using Cramer's rule for the two wall segment case. This is how the radiation exchange equations were derived in [3] and [5]. Cramer's rule is not a good numerical technique to use for the solution of linear systems (even for $2 \times 2$ systems) due to cancellation error that can be introduced when solving equations that are ill-conditioned.

\subsection{Algorithm for Calculating Four Wall Radiation Exchange}

The strategy for computing the radiation exchange between four wall segments is outlined below. RAD4 performs these steps directly or call subroutines that perform them. RAD2 and RAD10 follows the same logic.

Input

Temperatures Ceiling, Upper Wall, Lower Wall, Floor

\footnotetext{
${ }^{6}$ Gauss-Seidel or for example.
} 
Emissivities Ceiling, Wall, Floor

Absorptivities Upper, Lower Layer

Fire Size, Location, number

Room room number, dimensions, layer height

\section{Output}

Flux ceiling, upper wall, lower wall, floor

Energy Absorption Rate upper layer, lower layer

Steps 1. Calculate configuration factors, solid angles as described in section 2.2.2 and 2.1.6.

2. Determine the effective length between each pair of wall segments. From these lengths and inputted layer absorptivities calculate transmission factors for surface $\mathrm{j}$ to surface $\mathrm{k}$

3. Calculate transmission factors and gas layer absorptions for each fire $\mathrm{f}$ to surface $\mathrm{k}$ as outlined in section 2.1.7.

4. Calculate the energy absorbed by each gas layer due to upper/lower gas layer emission and due to the fire(s) following tables 2 and 3.

5. Set up the linear algebra

(a) Define vector $E$ using equation (24)

(b) Define matrix $\hat{A}$ using equation (25)

(c) Define matrix $B$ using equation (22)

(d) Define vector $c$, using equation (23) and Table 1.

6. Solve the linear system

$$
\hat{A} \Delta \hat{q}^{\prime \prime}=B E-c
$$

for $\Delta q^{\prime \prime}$ net radiation leaving each surface where $\Delta q^{\prime \prime}{ }_{k}=\Delta \hat{q}_{k}^{\prime \prime} \epsilon_{k}$. If the emittances are sufficiently close to 1 then use iteration to solve equation (27) otherwise use Gaussian elimination. The reason for this is due to the diagonal dominance of $\hat{A}$ as explained in the previous section.

7. Calculate the energy absorbed by the upper and lower gas layers due to the total energy , $q_{k}^{\text {out }}$ leaving each rectangle $k$.

\section{Computational Results}

\subsection{Checks}

Several simple checks can be made to verify a portion of the radiation calculation. First, no heat transfer occurs when all wall segments and both gas layers are at the same temperature. Therefore, the net radiation flux, $\Delta q^{\prime \prime}{ }_{k}$ given off by each surface and the energy absorbed by the gas should be zero under these uniform temperature conditions. Second, when there is no fire, the net energy absorbed by the gas must be the same as the net energy given off by the wall segments or equivalently 
Table 4: Four Wall Radiation Algorithm Timings

\begin{tabular}{|l||l|l|}
\hline Case & Total Time (s) & Linear Solve Time (s) \\
\hline $\begin{array}{l}45 \text { configuration factors } \\
\text { and direct linear solve }\end{array}$ & .2 & .06 \\
\hline $\begin{array}{l}\text { Eight configuration fac- } \\
\text { tors and iterative linear } \\
\text { solve }\end{array}$ & .06 & .01 \\
\hline $\begin{array}{l}\text { Two configuration fac- } \\
\text { tors and direct linear } \\
\text { solve }\end{array}$ & .012 & .001 \\
\hline
\end{tabular}

$$
q_{\text {lower }}+q_{\text {upper }}=\text { energy absorbed by interior gases }=\sum_{k=1}^{N} A_{k} \Delta q^{\prime \prime}{ }_{k}
$$

When the layers are transparent then the above equation sums to zero even though the individual wall fluxes $\Delta q^{\prime \prime}{ }_{k}$ will in general be non-zero. The gas absorbance terms, $q_{\text {lower }}$ and $q_{\text {upper }}$, are computed by RAD2, RAD4 and RAD10. These values can be summed to verify that above equation is satisfied. These checks were found to be useful since they all failed to hold during some point in the development process!

\subsection{Timings}

Configuration calculations are one of the major bottle necks in the radiation exchange calculation. Techniques to reduce the number of these calculations will improve the algorithms efficiency. The original version of RAD4 was based on RAD10, a ten wall segment model. It computed 45 configuration factors directly. Subsequent versions of RAD4 computed eight and two configuration factors directly. Table 4 summarizes the time required by these three different versions of RAD4. This table shows that the first version of RAD4 used approximately $70 \%$ of the time to setting up the linear system and $30 \%$ solving it. Reducing the setup overhead by computing fewer configurations factors reduced the computation time required by a factor of 17 .

Why quibble over the timings of a subroutine that only took .2 seconds to execute to begin with? The relative impact of RAD4 on CFASTs performance can be measured by comparing the time required to execute DSOURC ${ }^{7}$ with and without RAD4. For a six room CFAST test case DSOURC took about .06 seconds $^{8}$. A routine that takes .2 seconds per room used in each room will result in a 21 -fold ${ }^{9}$ increase in computer time. Even the fastest version of RAD4 will cause an increase of execution time of 2.25 if it is used in each room.

\footnotetext{
${ }^{7}$ DSOURC is the subroutine CFAST uses to calculate the right hand side of the modeling differential equations. Most of the work is performed by this routine or routines that DSOURC calls.

${ }^{8}$ All times are measured on a Compaq 386/20 Deskpro. This computer has a $20 \mathrm{mhz}$ clock and uses a floating point accelerator (math co-processor). The actual times will be different on different computers. But the relative times and hence the conclusions should be the same.

${ }^{9}$ DSOURC time with RAD4/DSOURC time withoutRAD4 $=(.2 * 6+.06) / .06 \approx 21$
} 


\subsection{Comparisons of RAD2 with RAD4}

This subsection compares the predictions of a two wall radiation exchange model, RAD2, with a four-wall model, RAD4. One of the assumptions made in section 2.1.1 about $\mathrm{N}$ wall segment radiation models is that the temperature distribution of each wall segment is approximately uniform. The zone fire model CFAST models the temperature of four wall segments independently. Therefore, a two wall model for radiation exchange can break down when the temperatures of the ceiling and upper walls differ significantly. This could happen in CFAST, for example, when different wall materials are used to model the ceiling, walls and floor. To demonstrate this consider the following example.

To simplify the comparison between the two and four wall segment models, assume that the wall segments are black bodies (the emissivities of all wall segments are one) and the gas layers are transparent (the gas absorptivities are zero). This is legitimate since for this example we are only interested in comparing how a two wall and a four wall radiation algorithm transfer heat to wall segments. Let the room dimensions be $4 \times 4 \times 4$ $[\mathrm{m}]$, the temperature of the floor and the lower and upper walls be $300[\mathrm{~K}]$. Let the ceiling temperature vary from $300[\mathrm{~K}]$ to $600[\mathrm{~K}]$.

Figure 13 shows a plot of the heat flux striking the ceiling and upper wall as a function of the ceiling temperature. The two wall model predicts that the extended ceiling (a surface formed by combining the ceiling and upper wall into one wall segment) cools, while the four wall model predicts that the ceiling cools and the upper wall warms. The four-wall model moderates temperature differences that may exist between the ceiling and upper wall (or floor and lower wall) by allowing heat transfer to occur between the ceiling and upper wall. The two wall model is unable to predict heat transfer between the ceiling and the upper wall since it models them both as one wall segment.

A four-wall algorithm will also break down when the uniform temperature assumption is broken. This could occur when a fire is located nearer to one side of a room than another.

\section{Conclusions}

This report documented algorithms for computing radiative heat exchange for three special cases, a two-wall, four-wall and ten-wall model. The theoretical basis for a general $\mathrm{N}$ wall model is well documented in the literature $[7,8,9]$. But an implementation of an $\mathrm{N}$ wall model is not yet practical for a zone fire model due to the high computational costs compared to other components in a zone fire model. One step was taken towards making $\mathrm{N}$ wall models practical. For wall surfaces that are approximate black bodies $(\epsilon>.85)$ it was shown that the linear system of equations involving the unknown net radiative flux could be solved iteratively, reducing an o $\left(N^{3}\right)$ to an o $\left(N^{2}\right)$ problem. For specific cases, (four-wall, ten-wall) it was shown how to set up this linear system efficiently by avoiding unnecessary configuration factor calculations. For the general $\mathrm{N}$ wall problem it is not enough to solve the linear system efficiently. For example, in the ten-wall case the linear solve time is only $20 \%$ of the total solution time. Methods need to be found to calculate configuration factors more efficiently, perhaps at some cost in accuracy. 


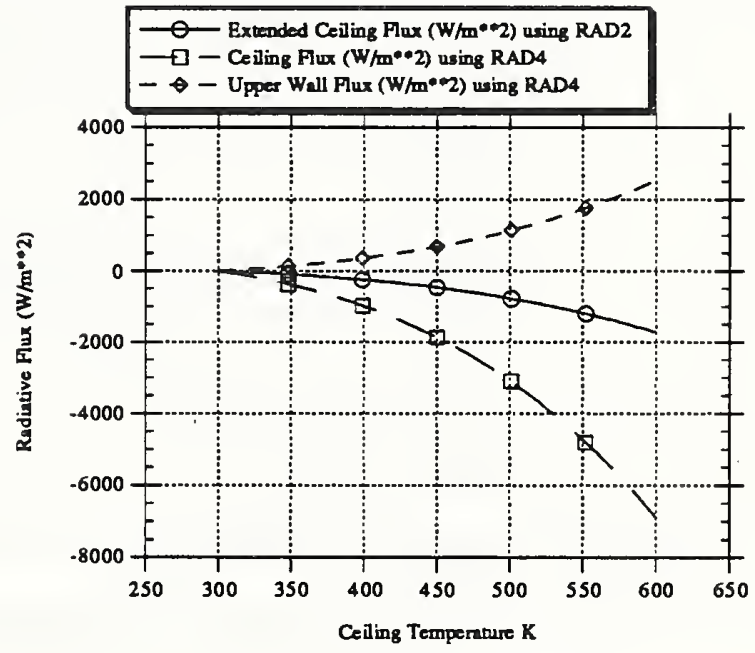

Figure 13: Ceiling, Upper Wall and Extended Ceiling Flux vs. Ceiling Temperature 


\section{Nomenclature}

A

A

A

$\hat{A}$

c

$B$

E

$D$

$F_{j-k}$

$N$

$N_{\text {fire }}$

$q$

$\Delta q^{\prime \prime}$

$T$

$\alpha$

$\delta$

$\epsilon$

$\chi$

$\phi$

$\pi$ area $\left[\mathrm{m}^{2}\right]$

absorption coefficient $\left[\mathrm{m}^{-1}\right]$

coefficient matrix for the net radiation equation.

coefficient matrix for diagonally dominant version of the net radiation equation

vector of source terms used in the net radiation equation (5) to represent energy contributions to wall segments due to gas emitting layers and point source fires [W]

matrix used on the right hand side of the net radiation equation

emmissive power, a vector whose $k^{\prime}$ th component is $\sigma T_{k}^{4}$

diagonal scaling matrix used to convert $A$ to the diagonally dominant version $\hat{A}$

geometric configuration factor, also called a view factor. The fraction of energy leaving a wall segment $j$ intercepted by wall segment $k$.

number of wall segments

number of fires

energy per unit time [W]

net heat flux leaving a wall segment $\left[\mathrm{W} / \mathrm{m}^{2}\right]$

temperature [K]

absorbance

dirac delta function; $\delta_{i j}=\left\{\begin{array}{cc}0 & \text { if } i \neq j \\ 1 & \text { if } i=j\end{array}\right.$

emittance, fraction of the black body radiation emitted by a gray surface or gray gas.

fraction of a fire's energy release rate that contributes to radiative heat transfer.

configuration factor

universal constant 


\section{Subscripts}

$j, k$
$j-k$
$f$
$f-k$
par
perp
total

\section{Superscripts}

"

fire

in

out

L

U

$L$, gas transmittance

average transmission factor where the average is computed over all possible paths between two wall segments

Stefan-Boltzmann constant, $\sigma=5.67 \times 10^{-8} \frac{\mathrm{W}}{\mathrm{m}^{2} \mathrm{~K}^{4}}$

reflectance, fraction of the energy reflected by a surface. This fraction can be related to the emittance, $\epsilon$, for a gray surface via $\epsilon=1-\rho$.

transmittance, fraction of the energy passing through a gas unimpeded. If the gas has uniform absorbency properties then $\tau$ can be computed using the Beer-Lambert law via $e^{-A L}$ where $\mathrm{A}$ is the absorbency of the gas per unit length and $\mathrm{L}$ is the length of the path through the gas.

a solid angle; The energy fraction of the f'th fire striking the k'th wall segment.

wall segments $j$ or $k$

from wall segment $j$ to wall segment $k$

fire $\mathbf{f}$

from fire $f$ to wall segment $k$

parallel rectangles which are identical and opposite

perpindicular rectangles which share a common edge

total energy release rate of a fire

flux, a quantity per unit area

a quantity due to a fire

incoming

outgoing

lower gas layer

upper gas layer

a quantity due to the lower gas layer 
a quantity due to the upper gas layer

$i, g a s$

a quantity due to the $i$ 'th gas layer where $i$ can be $L$ for lower or $U$ for upper layer 


\section{References}

[1] Henri E. Mitler and Howard W. Emmons. Documentation for $\mathrm{cfc} v$, the fifth harvard comptuer fire code. NBS-GCR 344, National Institute of Standards and Technology, 1981.

[2] Henri E. Mitler and John A. Rockett. User's guide to first, a comprehensive single-room fire model. Internal Report 3595, National Institute of Standards and Technology, 1987.

[3] T. Tanaka. A model of multiroom fire spread. Internal Report 2718, National Bureau of Standards, 1983.

[4] T. Tanaka. A model of multiroom fire spread. Fire Science and Technology, 3:105-121, 1983.

[5] Walter W. Jones and Richard D. Peacock. Technical reference guide for FAST version 18. Technical Note 1262, National Institute of Standards and Technology, 1989.

[6] Mark A. Dietenberger. Technical reference and user's guide for fast/ffm version 3. GCR 589, National Institute of Standards and Technology, 1991.

[7] Robert Siegel and John R. Howell. Thermal Radiation Heat Transfer. Hemisphere Publishing Corporation, New York, second edition, 1981.

[8] H. C. Hottel. Heat Transmission, chapter four. McGraw-Hill Book Company, New York, third edition, 1954.

[9] H.C. Hottel and E.S. Cohen. Radiant heat exchange in a gas filled enclosure: Allowance for non-uniformity of gas temperture. A I Ch E J, 4, 1958.

[10] Tokiyoshi. Yamada and Leonard Y. Cooper. Algorithms for calculating radiative heat exchange between the surfaces of an enclosure, the smoke layers and a fire, 1990. Unpublished slides from a talk given as a Center for Fire Research Colloquium, July 20, 1990 .

[11] Tokiyoshi Yamada. Algorithms for calculating radiative heat exchange between enclosure surfaces filled with smoke and a heat source, part i physical basis. Draft v.01, 1990 .

[12] Frank P. Incropera and David P. De Witt. Fundamentals of Heat and Mass Transfer. John Wiley and Sons, New York, third edition, 1990.

[13] Dougal Drysdale. An Introduction to Fire Dynamics. John Wiley and Sons, New York, 1985.

[14] A. T. Modak. Radiation from products of combustion, FMRC No. OAOE6.BU-1. Technical report, Factory Mutual Corporation, 1978.

[15] J. Quintiere. A perspective on compartment fire growth. Combustion Science and Technology, 39:11-54, 1984.

[16] J. Stoer and R. Bulirsch. Introduction to Numerical Analysis. Springer-Verlag, New York, 1980. 
[17] J. J. Dongarra, C. B. Moler, J. R. Bunch, and G. W. Stewart. LINPACK User's Guide. Society for Industrial and Applied Mathematics, Philadelphia, 1979.

[18] Glenn P. Forney and Walter W. Jones. Software development tools. Internal Report 4363, National Institute of Standards and Technology, 1990.

[19] J. Demmel, J. Dongarra, J. D. Croz, S. Hammarling A. Greenbaum, and D. Sorensen. Prospectus for the development of a linear algebra library for high-performance computers. Tech. Rep. TM-97, Mathematics and Computer Science Div., Argonne National Laboratory, Argonne, IL, 1987. 


\section{A Software Documentation}

This section shows how to use the radiation subroutines: RAD2, RAD4 and RAD10 in a zone fire model. They model the radiation exchange between two, four and ten wall segments in an enclosure respectively. These routines were designed to be compatible with the zone fire model CFAST. However, since these subroutines communicate with the calling routine via the argument list and not through common blocks it should be easy to incorporate them into other zone fire models. They calculate the energy absorbed by the gas layers and the heat flux striking the wall segments.

Linkage between the subroutines used by RAD2, RAD4 is represented by a computer generated text file given in Appendix B. RAD2, RAD4 and RAD10 were designed to be modular so that they can use many of the same routines. This simplifies debugging and shortens development time.

RAD2, RAD4 and RAD10 require wall properties such as geometric specifications, emittances and temperatures; gas properties such as absorptivities, layer height and temperatures. They calculate the rate of energy absorbed by the gas and the radiant heat flux striking the wall segment.

DEFFIGS, LAYFIG, RMFIG, PRPFIG2 and PARFIG2 calculate the configuration factors for RAD10. RAD2 and RAD4 use only RDPARFIG. The subroutine LAYFIG and RMFIG implement the configuration factor formulas found in section 3.1. LAYFIG calculates the configuration factors between the following 10 wall segments: ceiling, 4 upper wall segments, 4 lower wall segments and the floor. LAYFIG is called each time RAD10 is called. RMFIG on the other hand is called only once per run. It calculates the configuration factors between the ceiling, four walls and floor.

Transmission factors are computed by the subroutine DEFTAU and DEFTAUF. DEFTAU computes transmission factors between wall surfaces and DEFTAUF computes transmission factors between wall surfaces and fires. The beam length chosen is based upon a path between the center of two rectangle.

The linear system in equation (19) is solved directly or iteratively depending on how close the emittances are to 1 or equivalently how close the wall surfaces are to a black body. Routines SGEFA and SGESL are used to solve the linear system directly. They are a part of Linpack [17]. For the 10 wall case, a simple iterative scheme based on the GaussSeidel algorithm is used to solve the linear system when the emittances values indicate that convergence can be achieved with few iterations. This is not necessary for the two and four-wall cases since the speed up would be inconsequential.

\section{A.1 Using Subroutines RAD4 and RAD2}

Subroutines RAD2 and RAD4 calculate the radiation exchange between two wall segments and four wall segments respectively. RAD2 and RAD4 have identical interfaces. Either routine may be used in place of the other without changing code in the calling routine. RAD2 returns the same flux for the lower wall and the floor. The following FORTRAN routine is an example of how to use RAD2 and RAD4. This routine was used to generate the data plotted in figure 13 .

PROGRAM MAIN

INCLUDE 'PRECIS. INC'

DIMENSION THALL(4), TLAY (2), EMIS (4), ABSORB (2)

DIMENSION QFLUX(4), QLAY(2) 


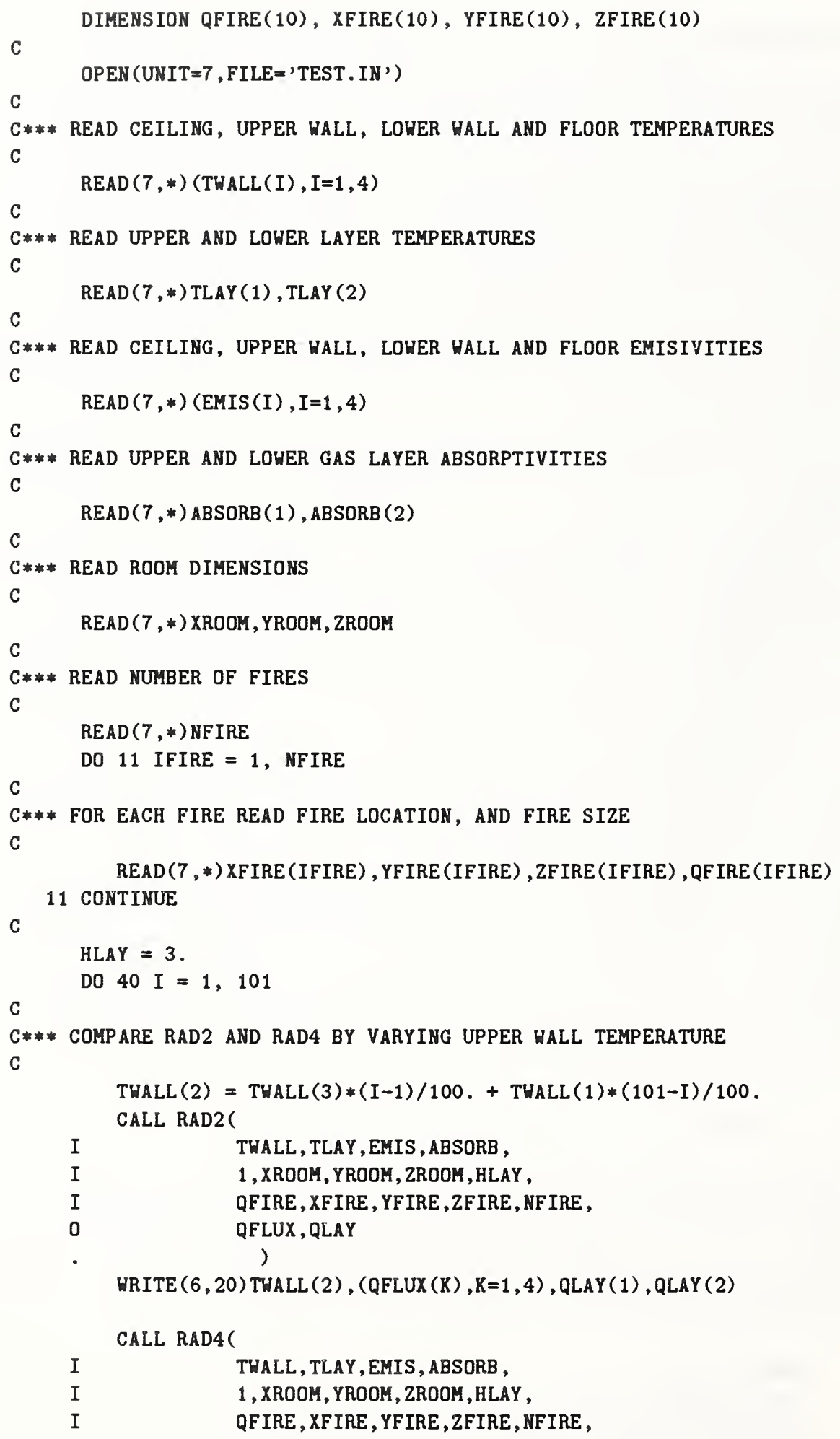




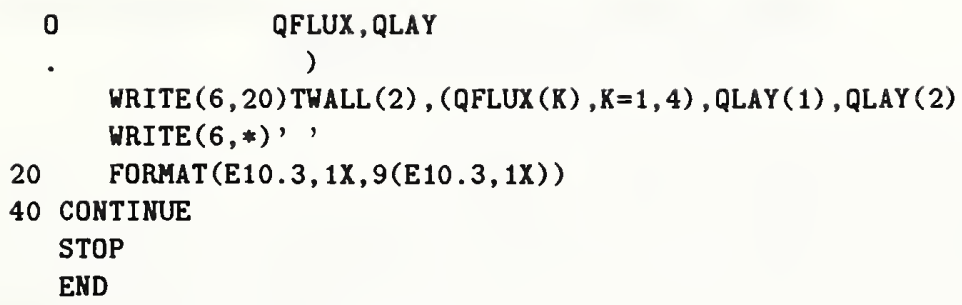

A description of the inputs follow.

RAD2, RAD4 Interface INPUTS

TWALL

TLAY

EMIS

ABSORB

XROOM

YROOM

ZROOM

HLAY

QFIRE

XFIRE

YFIRE

ZFIRE

\section{OUTPUTS}

TWALL(I) is the temperature of the I'th surface [K] where $I=1,2,3,4$ denotes the ceiling, the upper wall, the lower wall and the floor respectively

TLAY $(\mathrm{I})$ is the temperature of the I'th layer $[\mathrm{K}]$ where $\mathrm{I}=1,2$ denotes the upper and lower layers respectively

EMIS(I) is the emittance of the ceiling ( $\mathrm{I}=1)$, upper and lower walls $(I=2,3)$ and floor $(I=4)$

ABSORB(I) is the absorptance $\left[m^{-1}\right]$ of the upper $(I=1)$ and lower layer $(I=2)$

$\mathrm{XROOM}$ is the size of the room $[\mathrm{m}]$ in the $\mathrm{x}^{\prime}$ th coordinate direction.

YROOM is the size of the room [m] in the y'th coordinate direction.

ZROOM is the size of the room $[\mathrm{m}]$ in the z'th (vertical) coordinate direction.

HLAY is the height of the smoke layer interface above the floor [m]

QFIRE is an array of length NFIRE; QFIRE(IFIRE) is the energy release rate due to radiation of the IFIRE'th fire [W]

XFIRE is the $\mathrm{x}$ coordinate of the fire location [m]

YFIRE is the y coordinate of the fire location [m]

XFIRE is the $\mathrm{z}$ (vertical) coordinate of the fire location [m]
QFLUX

QFLUX(I) is the radiant heat flux $\left[\mathrm{W} / \mathrm{m}^{2}\right]$ to the I'th surfaces where $i=1,2,3,4$ denotes the ceiling, the upper wall, the lower wall and the floor respectively 


\section{A.2 Using the Subroutine RAD10}

The interface for RAD10 is essentially the same as the interface for RAD2 and RAD4. The variables TLAY, EMIS and QFLUX are arrays of size 4 for RAD2 and RAD4. In RAD10, the arrays TLAY, EMIS and QFLUX are dimensioned as size 10 rather than size 4, corresponding in order, to the 10 wall segments: ceiling, upper front, upper right, upper back, upper left, lower front, lower right, lower back, lower left walls and floor. RAD10 treats these wall segments independently.

\section{B Radiation Package Subroutine Structure - Who Calls Whom}

This appendix contains information on the subroutines used by RAD2, RAD4 and RAD10 are related to each other. This information is provided to make it easier to understand its structure. Each entry has up to four subheadings: CALLS, LIB, COMMONS and CALLED BY. The subheadings CALLS and LIB are similar. They both list external references to NAME., i.e., routines that NAME calls. The source code for the routines that are listed under CALLS appear in the same computer file as NAME. On the other hand, the routines listed by the LIB sub-heading do not. Some examples of routines that would appear under the LIB heading are FORTRAN supplied functions such as ABS, SQRT, MODE, etc. The radiation routines documented here do not have common blocks so there will be no information under this heading. The routines that are listed next to CALLED BY are those routines that call NAME. This appendix was generated by a computer program named ROADMAP documented in [18].

NAME

CALLS: $\quad$ SUB1, SUB2, $\ldots$

LIB: $\quad$ SUBA, SUBB, ...

COMMONS: COM1, COM2, .

CALLED BY: 'SUBa, SUBb, ...

SOURCE ROUTINES

ROUTINE: ISAMAX

LIB: $\quad$ ABS

CALLED BY: SGEFA

ROUTINE: RAD2 
CALLS: RDABS RDFLUX RDPARFIG RDSANG

LIB : $\quad$ EXP

CALLED BY: NONE - NO ROUTINES CALL RAD2

ROUTINE : RAD4

CALLS: RDABS RDFANG RDFLUX RDFTRAN RDPARFIG

RDRTRAN SDOT

SGEFA SGESL

LIB: $\quad$ SQRT

CALLED BY: NONE - NO ROUTINES CALL RAD4

ROUTINE: RDABS

CALLED BY: RAD2 RAD4

ROUTINE: RDFANG

CALLS: RDSANG

CALLED BY: RAD4

ROUTINE : RDFLUX

CALLED BY: RAD2 RAD4

ROUTINE: RDFTRAN

LIB : ABS EXP

CALLED BY: RAD4

ROUTINE: RDPARFIG

LIB : ATAN LOG SQRT

CALLED BY: RAD2 RAD4

ROUTINE: RDPRPFIG

LIB : ATAN LOG SQRT

CALLED BY: NONE - NO ROUTINES CALL RDPRPFIG

ROUTINE: RDRTRAN

LIB : EXP

CALLED BY: RAD4

ROUTINE: RDSANG

CALLS: RDSANG1

LIB : ABS SIGN

CALLED BY: RAD2 RDFANG

ROUTINE: RDSANG 1

LIB : ASIN SQRT

CALLED BY: RDSANG

ROUTINE: SAXPY

LIB : MOD

CALLED BY: SGEFA SGESL

ROUTINE : SDOT

CALLS : $\quad$ SDOT 
$\begin{array}{llll}\text { LIB : } & \text { MOD } & & \\ \text { CALLED BY : } & \text { RAD4 } & \text { SDOT } & \text { SGESL }\end{array}$

ROUTINE: SGEFA

CALLS: ISAMAX SAXPY SSCAL

CALLED BY: RAD4

ROUTINE : SGESL

CALLS: SAXPY SDOT

CALLED BY: RAD4

ROUTINE : SNRM2

LIB : ABS DBLE SQRT

CALLED BY: NONE - NO ROUTINES CALL SNRM2

ROUTINE: SSCAL

LIB: MOD

CALLED BY: SGEFA

\section{Subroutine Glossary}

The following is a glossary of subroutines used by the radiation packages RAD2, RAD4 and RAD10.

BLAS A collection basic linear algebra subroutines used by the linpack subroutines SGEFA and SGESL to solve the net radiation linear system of equations. These routines are documented in [19].

LINPACK The routines SGEFA and SGESL from LINPACK are used to factor and solve the net radiation linear system of equations. These routines are documented in [17].

ITER

This routine solves the linear system,

$$
A x=b
$$

iteratively by noting the fact that $A=I-G$ where

$$
\begin{aligned}
\|G\| & <1 \\
(I-G)^{-1} & =I+G+G^{2}+G^{3}+\cdots
\end{aligned}
$$

so that

$$
x=\left(I+G+G^{2}+\ldots\right) b
$$

This routine is only designed for use with RAD10. Note that $G^{N}$ is not explicitly formed. Only a few iterations are required when the emittances are close to 1 .

LAYFIG This routine calculates the configuration factors for surfaces in a rectangular box with a layer interface. This routine needs to be called each time the layer height changes. 
RAD2

RAD4

RAD10

RDABS

RDFTRAN

RDRTRAN

RDFLUX

RDPARFIG

RDPRPFIG

RDSANG

RDSANG1

RMFIG
This routine computes the radiative heat flux to the extended ceiling (ceiling + upper wall) and the extended floor (floor + lower wall) due to a point source fire, emitting absorbing gas layers (upper and lower) and heat emitting wall segments. It also computes the heat absorbed by the lower and upper layers.

This routine computes the radiative heat flux to the ceiling, upper wall, lower wall and floor due to a point source fire, emitting absorbing gas layers (upper and lower) and heat emitting wall segments. This routine also computes the heat absorbed by the lower and upper layers.

This routine computes the radiative heat flux to the ceiling, four upper walls (upper front, upper right, upper back and upper left), four lower walls (lower front, lower right, lower back and lower left), due to a point source fire(s), emitting/absorbing gas layers (upper and lower) and heat emitting wall segments. This routine also computes the heat absorbed by the lower and upper layers. It performs the same calculations as RAD4 and RAD2 except for the number of wall segments considered.

This routine computes the energy absorbed by the upper and lower layer due to radiation given off by heat emitting rectangles forming the enclosure. This energy absorption is added to the variables, QLLAY and QULAY which were previously used to contain the heat absorbed by the lower and upper layers due to gas emissions and fires.

This routine calculates the transmissivities, $\tau_{f-k}^{U}$ and $\tau_{f-k}^{L}$ between each fire $f$ and each wall segment $k$.

This routine calculates the transmissivities, $\tau_{j-k}^{U}$ and $\tau_{j-k}^{L}$ between each pair of wall segments $j$ and $k$.

This routine calculates the ' $c$ ' vector in the net radiation equations of Siegel and Howell and the enthalpy absorbed by the lower and upper layer fires due to gas layer emission and fires.

This routine calculates the configuration factor between two identical parallel rectangles.

This routine calculates the configuration factor between two perpendicular rectangles that share a common edge.

This routine computes the solid angle of an arbitrary rectangle.

This routine computes the solid angle of a rectangle whose corner is tangent to the surface of a sphere. The center of this sphere is the location of the point source fire.

This routine calculates the configuration factors for surfaces in a rectangular box. This calculation needs to be done only once per room, 
assuming that the room does not change size during the simulation. 


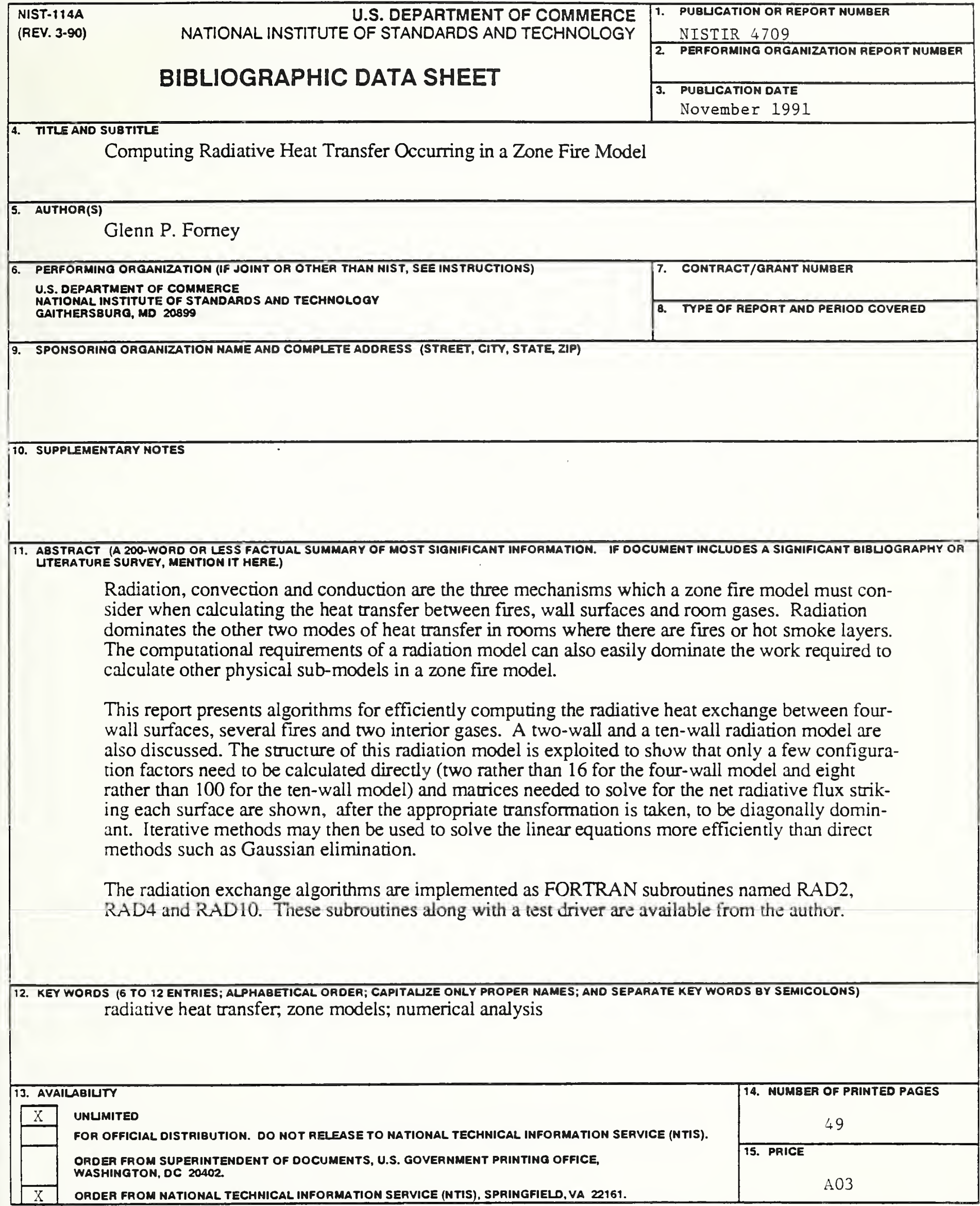

ELECTRONIC FORM 


